\title{
Decentralized Robust Adaptive Output Feedback Stabilization for Interconnected Nonlinear Systems with Uncertainties
}

\author{
Qiang Yang, ${ }^{1,2}$ Ming Zhu, ${ }^{1}$ Tao Jiang, ${ }^{1}$ Jin He, ${ }^{1}$ Jianying Yuan, ${ }^{1}$ and Jianda Han ${ }^{2}$ \\ ${ }^{1}$ School of Control Engineering, Chengdu University of Information Technology, Chengdu, Sichuan 610225, China \\ ${ }^{2}$ State Key Laboratory of Robotics, Shenyang Institute of Automation, Chinese Academy of Sciences, Liaoning 100190, China \\ Correspondence should be addressed to Ming Zhu; zhuming@cuit.edu.cn
}

Received 2 May 2016; Accepted 11 July 2016

Academic Editor: Ning Cai

Copyright (c) 2016 Qiang Yang et al. This is an open access article distributed under the Creative Commons Attribution License, which permits unrestricted use, distribution, and reproduction in any medium, provided the original work is properly cited.

\begin{abstract}
Based on adaptive nonlinear damping, a novel decentralized robust adaptive output feedback stabilization comprising a decentralized robust adaptive output feedback controller and a decentralized robust adaptive observer is proposed for a largescale interconnected nonlinear system with general uncertainties, such as unknown nonlinear parameters, bounded disturbances, unknown nonlinearities, unmodeled dynamics, and unknown interconnections, which are nonlinear function of not only states and outputs but also unmodeled dynamics coming from other subsystems. In each subsystem, the proposed stabilization only has two adaptive parameters, and it is not needed to generate an additional dynamic signal or estimate the unknown parameters. Under certain assumptions, the proposed scheme guarantees that all the dynamic signals in the interconnected nonlinear system are bounded. Furthermore, the system states and estimate errors can approach arbitrarily small values by choosing the design parameters appropriately large. Finally, simulation results illustrated the effectiveness of the proposed scheme.
\end{abstract}

\section{Introduction}

Various practical systems, such as ecosystems, economic systems, transportation systems, and power systems, are examples of large-scale nonlinear systems. Generally speaking, a large-scale nonlinear system comprises several subsystems with many interconnections. It is impossible to incorporate many feedback loops into the centralized controller and it is too costly even if they can be implemented. To overcome the limitations of the traditional centralized control that requires sufficiently large communications bandwidth to exchange information between subsystems, many researchers developed decentralized control, which uses local information available in each subsystem only, for large-scale interconnected nonlinear systems [1-16]. Particularly, based on the linear quadratic design, a sufficient condition was presented for existence of the decentralized output feedback stabilization for a class of nonlinear interconnected systems [1], and the estimation of the associated stability domain was also given. In [6], based on local Luenberger-type observers, a decentralized robust dynamic output feedback stabilization scheme was proposed for a class of nonlinear interconnected systems, which are composed of linear subsystems coupled by nonlinear time-varying interconnections satisfying quadratic constraints. In [7], based on linear matrix inequalities, decentralized robust stabilization scheme was designed for a class of nonlinear interconnected systems, where each subsystem is composed of a linear part and an uncertain additive nonlinearity which is a discontinuous function of time and state of the overall system. Based on neural networks, decentralized robust stabilization scheme was proposed for large-scale nonlinear interconnected systems [10, 11, 16]. Based on adaptive nonlinear damping [3], a decentralized robust adaptive control was proposed for a class of largescale nonlinear systems with unmodeled dynamics, where an additional dynamic signal was introduced in each subsystem to dominate the unmodeled dynamics.

Many important results have been obtained by the efforts of researchers for large-scale interconnected nonlinear systems stabilization. However, there are still many unresolved 
issues. On the one hand, general uncertainties should be considered in the subsystems. Particularly, in addition to uncertain interconnections, unmodeled dynamics and uncertain nonlinearities in the subsystems were not considered in $[1,2$, 6-9]. In [10, 11, 13-15], uncertain interconnections and model nonlinearities were considered; the unmodeled dynamics still were not considered in the subsystems. In [3], unmodeled dynamics were considered, but an additional dynamic signal should be generated in the proposed scheme using some information about the unmodeled dynamics, which are often not available in practice. It is worth noting that, with regard to the interconnections, researchers mainly considered the situation that the interconnections are function of states or outputs coming from other subsystems; almost no one considered the situation in which the interconnections including unmodeled dynamics are coming from other subsystems but which may exist in practice. On the other hand, most of the observers used in the above mentioned literatures are not robust adaptive observer; for example, Luenbergertype observer was used in [6,7], sliding mode observer was used in [9], and high gain observer was used in [4, 10]. However, Luenberger-type observer has poor robustness to the system uncertainties [17]. Sliding mode observer and high gain observer may exhibit oscillating or peaking phenomenon in the transient behavior, which will adversely affect the performance of the closed-loop system. Robust adaptive observer can well overcome these problems. So, a decentralized robust adaptive output feedback stabilization with a decentralized robust adaptive observer for a large-scale interconnected nonlinear system in the presence of general uncertainties in each subsystem is meaningful.

In this paper, a novel decentralized robust adaptive output feedback stabilization, which is composed of a decentralized robust adaptive output feedback controller and a decentralized robust adaptive observer, is proposed for a large-scale interconnected nonlinear system with general uncertainties, such as unknown nonlinear parameters, bounded disturbances, unknown nonlinearities, unmodeled dynamics, and unknown interconnections. The unknown interconnections are nonlinear function of not only states and outputs but also unmodeled dynamics coming from other subsystems. Comparing with the aforementioned literatures, it is the first time that unmodeled dynamics coming from the other subsystems are considered in the uncertain interconnections.

Based on adaptive nonlinear damping, the proposed stabilization only has two adaptive parameters in each subsystem, and it is not needed to generate an additional dynamic signal or estimate the unknown parameters. Under certain assumptions, the proposed scheme guarantees that all the dynamic signals in the interconnected system are bounded. Furthermore, the system states and estimate errors can approach arbitrarily small values by choosing the design parameters appropriately large. Finally, simulation results illustrated the effectiveness of the proposed scheme.

\section{Problem Statement}

Consider a large-scale interconnected nonlinear system composed of $N$ subsystems.
The $i$ th subsystem is given as

$$
\begin{aligned}
\dot{x}_{i} & =A_{i} x_{i}+B_{i}\left[f_{i}\left(x_{i}, \omega_{i}, \theta_{i}, d_{i}\right)+u_{i}\right. \\
& \left.+\sum_{j=1, j \neq i}^{N} H_{i j}\left(x_{j}, y_{j}, \omega_{j}, \theta_{j}\right)\right], \\
y_{i} & =C_{i} x_{i},
\end{aligned}
$$

where, for each $i=1,2, \ldots, N, x_{i} \in R^{n_{i}}, y_{i} \in R^{l_{i}}$, and $u_{i} \in R^{m_{i}}$ are the state, the output, and the control input of the $i$ th subsystem, respectively; $\omega_{i} \in R^{r_{i}}$ are the unmodeled dynamics; $\theta_{i} \in R^{q_{i}}$ are the unknown parameters; $d_{i} \in R^{k_{i}}$ are the unknown bounded disturbances; $f_{i}\left(x_{i}, \omega_{i}, \theta_{i}, d_{i}\right) \in R^{m_{i}}$ is an unknown nonlinear function vector representing the uncertainties in the $i$ th subsystem; $H_{i j}\left(x_{j}, y_{j}, \omega_{j}, \theta_{j}\right) \in R^{m_{i}}$, where $j=1,2, \ldots, N$ and $j \neq i$, is an unknown nonlinear function vector representing the uncertain interconnections between the $i$ th subsystem and the $j$ th one; $A_{i}, B_{i}$, and $C_{i}$ are known constant matrices with appropriate dimensions. Without loss of generality, we assumed that zero is the only equilibrium point for the system.

The objective is to design a decentralized robust adaptive output feedback stabilization, which includes a decentralized robust adaptive controller and a decentralized robust adaptive observer, for large-scale interconnected nonlinear system (1) in the presence of general uncertainties. We need the following assumptions.

Assumption 1. For each subsystem, there exist unknown constants $\rho_{i 1} \geq 0$ and $\rho_{i 2} \geq 0$, such that the unmodeled dynamics satisfy

$$
\left\|\omega_{i}(t)\right\| \leq \rho_{i 1}+\rho_{i 2}\left\|x_{i}(t)\right\|,
$$

where $x_{i} \in R^{n_{i}}$ is the state in the $i$ th subsystem.

Remark 2. On the one hand, unmodeled dynamics widely appear in practical nonlinear system. It is not sagacious if we ignore the effect of the unmodeled dynamics on the entire system. On the other hand, the effect of unmodeled dynamics on the practical nonlinear system should be a limited one. Otherwise, it should be modeled. As Assumption 1 implies, the norm of the unmodeled dynamics should be constrained by the norm of the modeled dynamics. Comparing with the assumption to the unmodeled dynamics in [18], which is exponentially input-to-state practically stable, much fewer messages of the unmodeled dynamics are needed in this paper.

Assumption 3. For each subsystem, there exist unknown constants $c_{i k} \geq 0, k=1,2, \ldots, 8$, such that

$$
\begin{aligned}
\left\|f_{i}\left(x_{i}, \omega_{i}, \theta_{i}, d_{i}\right)\right\| \leq & c_{i 1}+c_{i 2}\left\|x_{i}\right\| \varphi_{i}\left(y_{i}\right) \\
& +c_{i 3}\left\|\omega_{i}\right\| \psi_{i}\left(y_{i}\right)+c_{i 4} \varsigma_{i}\left(y_{i}\right),
\end{aligned}
$$




$$
\begin{gathered}
\left\|H_{i j}\left(x_{j}, y_{j}, \omega_{j}, \theta_{j}\right)\right\| \leq c_{i 5}+c_{i 6}\left\|x_{j}\right\|+c_{i 7}\left\|y_{j}\right\| \\
+c_{i 8}\left\|\omega_{j}\right\|,
\end{gathered}
$$

where $\varphi_{i}\left(y_{i}\right), \psi_{i}\left(y_{i}\right)$, and $\varsigma_{i}\left(y_{i}\right)$ are known nonnegative nonlinear functions.

Remark 4. From Assumption 3, we can see that the uncertainties studied in the $i$ th subsystem are very general. In addition to $y_{i}$, unmeasured signals $x_{i}, \omega_{i}, x_{j}, y_{j}$, and $\omega_{j}$ are contained in the right sides of (3). The unknown nonlinear function $H_{i j}\left(x_{j}, y_{j}, \omega_{j}, \theta_{j}\right)$ satisfies the Lipschitz condition, but the Lipschitz condition is a special case for the unknown nonlinear function $f_{i}\left(x_{i}, \omega_{i}, \theta_{i}, d_{i}\right)$. Similar assumptions can be seen in $[19,20]$. Furthermore, the uncertain interconnected terms $\sum_{j=1, j \neq i}^{N} H_{i j}\left(x_{j}, y_{j}, \omega_{j}, \theta_{j}\right)$ can be very strong since all the states $\|\mathbf{x}\|$, outputs $\|\mathbf{y}\|$, and unmodeled dynamics $\|\boldsymbol{\omega}\|$ coming from the other subsystems are considered and are not assumed to be bounded to a known or unknown constant.

Assumption 5. For each subsystem, $\left(A_{i}, B_{i}\right)$ is controllable and $\left(A_{i}, C_{i}\right)$ is detectable.

Assumption 6. For each subsystem, there exist positive-define matrices $\left(P_{i}, Q_{i}\right)$, such that

$$
\begin{aligned}
A_{m i}^{T} P_{i}+P_{i} A_{m i} & =-Q_{i}, \\
P_{i} B_{i} & =C_{i}^{T},
\end{aligned}
$$

where, choosing a matrix $L_{i}$, one lets $A_{m i}=A_{i}-B_{i} L_{i}$ be a strict Hurwitz matrix.

\section{Decentralized Robust Adaptive Output Feedback Stabilization}

In this paper, based on adaptive nonlinear damping, the proposed robust adaptive output feedback stabilization scheme composed of a decentralized robust adaptive output feedback controller and a decentralized robust adaptive observer for each subsystem is as follows.

Decentralized robust adaptive controller is

$$
\begin{aligned}
u_{i} & =-\beta_{i 1} C_{i} \hat{x}_{i} \beta_{m i 1}, \\
\dot{\beta}_{i 1} & =\left\|C_{i} \hat{x}_{i}\right\|^{2} \beta_{m i 1}-\beta_{i 1}+\beta_{i 1}^{*}, \\
\beta_{m i 1} & =1+\varsigma_{i}^{2}\left(y_{i}\right)+\varphi_{i}^{2}\left(y_{i}\right)+2 \psi_{i}^{2}\left(y_{i}\right) .
\end{aligned}
$$

Decentralized robust adaptive observer is

$$
\begin{gathered}
\dot{\hat{x}}_{i}=A_{m i} \widehat{x}_{i}+\beta_{i 2} B_{i}\left(y_{i}-C_{i} \widehat{x}_{i}\right) \beta_{m i 2}, \\
\dot{\beta}_{i 2}=\left\|y_{i}-C_{i} \hat{x}_{i}\right\|^{2} \beta_{m i 2}-\beta_{i 2}+\beta_{i 2}^{*}, \\
\beta_{m i 2}=1+c_{i}^{2}\left(y_{i}\right)+\varphi_{i}^{2}\left(y_{i}\right)+2 \psi_{i}^{2}\left(y_{i}\right) \\
+\left\|\beta_{i 1} C_{i} \widehat{x}_{i} \beta_{m i 1}\right\|^{2},
\end{gathered}
$$

where $\beta_{i 1}^{*}>0$ and $\beta_{i 2}^{*}>0$ are two design constants, which are the desired value of adaptive parameters $\beta_{i 1}$ and $\beta_{i 2}$, respectively; that is, when $\beta_{i 1}=\beta_{i 1}^{*}$ and $\beta_{i 2}=\beta_{i 2}^{*}$, the output feedback controller and observer have a desired performance. It will be shown that the bigger values of $\beta_{i 1}^{*}$ and $\beta_{i 2}^{*}$ are chosen, the smaller system states and estimation errors will be obtained.

Letting $e_{i}=x_{i}-\widehat{x}_{i}$, from (1) and (8), we have

$$
\begin{aligned}
\dot{e}_{i} & =A_{m i} e_{i}+B_{i}\left(L_{i} x_{i}+f_{i}\left(x_{i}, \omega_{i}, \theta_{i}, d_{i}\right)+u_{i}\right. \\
& \left.+\sum_{j=1, j \neq i}^{N} H_{i j}\left(x_{j}, y_{j}, \omega_{j}, \theta_{j}\right)\right)-\beta_{i 2} B_{i} \bar{e}_{i} \beta_{m i 2},
\end{aligned}
$$

where $\bar{e}_{i}=y_{i}-C_{i} \widehat{x}_{i}=C_{i} e_{i}$.

Theorem 7. If Assumptions 1-6 are satisfied, the decentralized robust adaptive output feedback stabilization given by (5)-(10) ensures that all the dynamic signals in large-scale interconnected nonlinear system (1) are uniformly bounded in the presence of uncertain interconnections, unknown nonlinear parameters, bounded disturbances, unknown nonlinearities, and unmodeled dynamics in each subsystem. Furthermore, choosing the design parameters $\beta_{i 1}^{*}$ and $\beta_{i 2}^{*}$ appropriately large, the system states and estimation errors can be made arbitrarily small.

Proof. Choose the Lyapunov function candidate

$$
\begin{aligned}
V= & \frac{1}{2} \\
& \cdot \sum_{i=1}^{N}\left[x_{i}^{T} P_{i} x_{i}+e_{i}^{T} P_{i} e_{i}+\left(\beta_{i 1}-\beta_{i 1}^{*}\right)^{2}+\left(\beta_{i 2}-\beta_{i 2}^{*}\right)^{2}\right] .
\end{aligned}
$$

Taking the derivative of $V$ gives

$$
\begin{aligned}
\dot{V} & =\sum_{i=1}^{N}\left\{-\frac{1}{2} x_{i}^{T} Q_{i} x_{i}+x_{i}^{T} P_{i} B_{i}\left(L_{i} x_{i}\right.\right. \\
& \left.+f_{i}\left(x_{i}, \omega_{i}, \theta_{i}, d_{i}\right)+u_{i}+\sum_{j=1, j \neq i}^{N} H_{i j}\left(x_{j}, y_{j}, \omega_{j}, \theta_{j}\right)\right) \\
& -\frac{1}{2} e_{i}^{T} Q_{i} e_{i}+e_{i}^{T} P_{i} B_{i}\left(L_{i} x_{i}+f_{i}\left(x_{i}, \omega_{i}, \theta_{i}, d_{i}\right)+u_{i}\right. \\
& \left.+\sum_{j=1, j \neq i}^{N} H_{i j}\left(x_{j}, y_{j}, \omega_{j}, \theta_{j}\right)\right)-\beta_{i 2} e_{i}^{T} P_{i} B_{i} \bar{e}_{i} \beta_{m i 2} \\
& +\left(\beta_{i 1}-\beta_{i 1}^{*}\right)\left\|C_{i} \widehat{x}_{i}\right\|^{2} \beta_{m i 1}-\left(\beta_{i 1}-\beta_{i 1}^{*}\right)^{2}+\left(\beta_{i 2}\right. \\
& \left.\left.-\beta_{i 2}^{*}\right)\left\|y_{i}-C_{i} \hat{x}_{i}\right\|^{2} \beta_{m i 2}-\left(\beta_{i 2}-\beta_{i 2}^{*}\right)^{2}\right\} .
\end{aligned}
$$


Since $x_{i}=\widehat{x}_{i}+e_{i}$, then

$$
\begin{aligned}
\dot{V} & =\sum_{i=1}^{N}\left\{-\frac{1}{2} x_{i}^{T} Q_{i} x_{i}+\widehat{x}_{i}^{T} P_{i} B_{i}\left(L_{i} x_{i}\right.\right. \\
& \left.+f_{i}\left(x_{i}, \omega_{i}, \theta_{i}, d_{i}\right)+u_{i}+\sum_{j=1, j \neq i}^{N} H_{i j}\left(x_{j}, y_{j}, \omega_{j}, \theta_{j}\right)\right) \\
& -\frac{1}{2} e_{i}^{T} Q_{i} e_{i}+2 e_{i}^{T} P_{i} B_{i}\left(L_{i} x_{i}+f_{i}\left(x_{i}, \omega_{i}, \theta_{i}, d_{i}\right)+u_{i}\right. \\
& \left.+\sum_{j=1, j \neq i}^{N} H_{i j}\left(x_{j}, y_{j}, \omega_{j}, \theta_{j}\right)\right)-\beta_{i 2} e_{i}^{T} P_{i} B_{i} \bar{e}_{i} \beta_{m i 2} \\
& +\left(\beta_{i 1}-\beta_{i 1}^{*}\right)\left\|C_{i} \hat{x}_{i}\right\|^{2} \beta_{m i 1}-\left(\beta_{i 1}-\beta_{i 1}^{*}\right)^{2}+\left(\beta_{i 2}\right. \\
& \left.\left.-\beta_{i 2}^{*}\right)\left\|y_{i}-C_{i} \hat{x}_{i}\right\|^{2} \beta_{m i 2}-\left(\beta_{i 2}-\beta_{i 2}^{*}\right)^{2}\right\} .
\end{aligned}
$$

Substituting (5) into (14) gives

$$
\begin{aligned}
\dot{V} & =\sum_{i=1}^{N}\left\{-\frac{1}{2} x_{i}^{T} Q_{i} x_{i}+\widehat{x}_{i}^{T} P_{i} B_{i}\left(L_{i} x_{i}\right.\right. \\
& \left.+f_{i}\left(x_{i}, \omega_{i}, \theta_{i}, d_{i}\right)+\sum_{j=1, j \neq i}^{N} H_{i j}\left(x_{j}, y_{j}, \omega_{j}, \theta_{j}\right)\right)-\frac{1}{2} \\
& \cdot e_{i}^{T} Q_{i} e_{i}+2 e_{i}^{T} P_{i} B_{i}\left(L_{i} x_{i}+f_{i}\left(x_{i}, \omega_{i}, \theta_{i}, d_{i}\right)+u_{i}\right. \\
& \left.+\sum_{j=1, j \neq i}^{N} H_{i j}\left(x_{j}, y_{j}, \omega_{j}, \theta_{j}\right)\right)-\beta_{i 1}^{*}\left\|C_{i} \widehat{x}_{i}\right\|^{2} \beta_{m i 1} \\
& -\beta_{i 2}^{*}\left\|y_{i}-C_{i} \hat{x}_{i}\right\|^{2} \beta_{m i 2}-\left(\beta_{i 1}-\beta_{i 1}^{*}\right)^{2}-\left(\beta_{i 2}\right. \\
& \left.\left.-\beta_{i 2}^{*}\right)^{2}\right\} .
\end{aligned}
$$

Substituting (3) into (15) gives

$$
\begin{aligned}
\dot{V} & \leq \sum_{i=1}^{N}\left\{-\frac{1}{2} x_{i}^{T} Q_{i} x_{i}+\left\|C_{i} \hat{x}_{i}\right\|\left[\left\|L_{i}\right\|\left\|x_{i}\right\|+c_{i 1}\right.\right. \\
& +c_{i 2}\left\|x_{i}\right\| \varphi_{i}\left(y_{i}\right)+c_{i 3}\left\|\omega_{i}\right\| \psi_{i}\left(y_{i}\right)+c_{i 4} \varsigma_{i}\left(y_{i}\right) \\
& \left.+\sum_{j=1}^{N}\left(c_{i 5}+c_{i 6}\left\|x_{j}\right\|+c_{i 7}\left\|y_{j}\right\|+c_{i 8}\left\|\omega_{j}\right\|\right)\right]-\frac{1}{2} \\
& \cdot e_{i}^{T} Q_{i} e_{i}+2\left\|\bar{e}_{i}\right\|\left[\left\|L_{i}\right\|\left\|x_{i}\right\|+c_{i 1}+c_{i 2}\left\|x_{i}\right\| \varphi_{i}\left(y_{i}\right)\right. \\
& +c_{i 3}\left\|\omega_{i}\right\| \psi_{i}\left(y_{i}\right)+c_{i 4} \varsigma_{i}\left(y_{i}\right)+\left\|u_{i}\right\|
\end{aligned}
$$

$$
\begin{aligned}
& \left.+\sum_{j=1}^{N}\left(c_{i 5}+c_{i 6}\left\|x_{j}\right\|+c_{i 7}\left\|y_{j}\right\|+c_{i 8}\left\|\omega_{j}\right\|\right)\right] \\
& -\beta_{i 1}^{*}\left\|C_{i} \widehat{x}_{i}\right\|^{2} \beta_{m i 1}-\beta_{i 2}^{*}\left\|y_{i}-C_{i} \widehat{x}_{i}\right\|^{2} \beta_{m i 2}-\left(\beta_{i 1}\right. \\
& \left.\left.-\beta_{i 1}^{*}\right)^{2}-\left(\beta_{i 2}-\beta_{i 2}^{*}\right)^{2}\right\} .
\end{aligned}
$$

Substituting (2) into (16) gives

$$
\begin{aligned}
\dot{V} \leq & \sum_{i=1}^{N}\left\{-\frac{1}{2} x_{i}^{T} Q_{i} x_{i}+\left\|C_{i} \hat{x}_{i}\right\|\left[\left\|L_{i}\right\|\left\|x_{i}\right\|+c_{i 1}\right.\right. \\
& +c_{i 2}\left\|x_{i}\right\| \varphi_{i}\left(y_{i}\right)+c_{i 3} \rho_{i 1} \psi_{i}\left(y_{i}\right)+c_{i 3} \rho_{i 2}\left\|x_{i}\right\| \psi_{i}\left(y_{i}\right) \\
& +c_{i 4} s_{i}\left(y_{i}\right) \\
& \left.+\sum_{j=1}^{N}\left(c_{i 5}+c_{i 6}\left\|x_{j}\right\|+c_{i 7}\left\|y_{j}\right\|+c_{i 8} \rho_{j 1}+c_{i 8} \rho_{j 2}\left\|x_{j}\right\|\right)\right] \\
& -\frac{1}{2} e_{i}^{T} Q_{i} e_{i}+2\left\|\bar{e}_{i}\right\|\left[\left\|L_{i}\right\|\left\|x_{i}\right\|+c_{i 1}+c_{i 2}\left\|x_{i}\right\| \varphi_{i}\left(y_{i}\right)\right. \\
& +c_{i 3} \rho_{i 1} \psi_{i}\left(y_{i}\right)+c_{i 3} \rho_{i 2}\left\|x_{i}\right\| \psi_{i}\left(y_{i}\right)+c_{i 4} \varsigma_{i}\left(y_{i}\right)+\left\|u_{i}\right\| \\
& \left.+\sum_{j=1}^{N}\left(c_{i 5}+c_{i 6}\left\|x_{j}\right\|+c_{i 7}\left\|y_{j}\right\|+c_{i 8} \rho_{j 1}+c_{i 8} \rho_{j 2}\left\|x_{j}\right\|\right)\right] \\
& -\beta_{i 1}^{*}\left\|C_{i} \widehat{x}_{i}\right\|^{2} \beta_{m i 1}-\beta_{i 2}^{*}\left\|y_{i}-C_{i} \hat{x}_{i}\right\|^{2} \beta_{m i 2}-\left(\beta_{i 1}\right. \\
& \left.\left.-\beta_{i 1}^{*}\right)^{2}-\left(\beta_{i 2}-\beta_{i 2}^{*}\right)^{2}\right\} .
\end{aligned}
$$

Since

$$
\begin{aligned}
& \sum_{j=1}^{N}\left(c_{i 5}+c_{i 6}\left\|x_{j}\right\|+c_{i 7}\left\|y_{j}\right\|+c_{i 8} \rho_{j 1}+c_{i 8} \rho_{j 2}\left\|x_{j}\right\|\right) \\
& \quad \leq N c_{i 5}+c_{i 8} \sum_{j=1}^{N} \rho_{j 1}+\sum_{j=1}^{N}\left(c_{i 6}+c_{i 7}\left\|C_{j}\right\|+c_{i 8} \rho_{j 2}\right)\left\|x_{j}\right\|
\end{aligned}
$$

taking

$$
\begin{aligned}
& \bar{c}_{i 1}=c_{i 1}+N c_{i 5}+c_{i 8} \sum_{j=1}^{N} \rho_{j 1}, \\
& \bar{c}_{i 6}=\max \left(c_{i 6}+c_{i 7}\left\|C_{j}\right\|+c_{i 8} \rho_{j 2}, j=1,2, \ldots, N\right)
\end{aligned}
$$

from (17)-(19), we have

$$
\begin{aligned}
\dot{V} & \leq \sum_{i=1}^{N}\left\{-\frac{1}{2} x_{i}^{T} Q_{i} x_{i}+\left\|C_{i} \hat{x}_{i}\right\|\left[\left\|L_{i}\right\|\left\|x_{i}\right\|+\bar{c}_{i 1}\right.\right. \\
& +c_{i 2}\left\|x_{i}\right\| \varphi_{i}\left(y_{i}\right)+c_{i 3} \rho_{i 1} \psi_{i}\left(y_{i}\right)+c_{i 3} \rho_{i 2}\left\|x_{i}\right\| \psi_{i}\left(y_{i}\right)
\end{aligned}
$$


Journal of Control Science and Engineering

5

$$
\begin{aligned}
& \left.+c_{i 4} \varsigma_{i}\left(y_{i}\right)+\bar{c}_{i 6} \sum_{j=1}^{N}\left\|x_{j}\right\|\right]-\frac{1}{2} e_{i}^{T} Q_{i} e_{i}+2\left\|\bar{e}_{i}\right\| \\
& \cdot\left[\left\|L_{i}\right\|\left\|x_{i}\right\|+\bar{c}_{i 1}+c_{i 2}\left\|x_{i}\right\| \varphi_{i}\left(y_{i}\right)+c_{i 3} \rho_{i 1} \psi_{i}\left(y_{i}\right)\right. \\
& +c_{i 3} \rho_{i 2}\left\|x_{i}\right\| \psi_{i}\left(y_{i}\right)+c_{i 4} \varsigma_{i}\left(y_{i}\right)+\left\|u_{i}\right\| \\
& \left.+\bar{c}_{i 6} \sum_{j=1}^{N}\left\|x_{j}\right\|\right]-\beta_{i 1}^{*}\left\|C_{i} \widehat{x}_{i}\right\|^{2} \beta_{m i 1}-\beta_{i 2}^{*}\left\|y_{i}-C_{i} \widehat{x}_{i}\right\|^{2} \\
& \left.\cdot \beta_{m i 2}-\left(\beta_{i 1}-\beta_{i 1}^{*}\right)^{2}-\left(\beta_{i 2}-\beta_{i 2}^{*}\right)^{2}\right\} .
\end{aligned}
$$

Since, for any $\Gamma>0, a b \leq(1 / 2)\left(\Gamma a^{2}+b^{2} / \Gamma\right)$, we have

$$
\begin{aligned}
& \left\|C_{i} \hat{x}_{i}\right\|\left\|L_{i}\right\|\left\|x_{i}\right\| \leq \frac{1}{2} \Gamma\left\|L_{i}\right\|^{2}\left\|C_{i} \hat{x}_{i}\right\|^{2}+\frac{1}{2 \Gamma}\left\|x_{i}\right\|^{2}, \\
& c_{i 2}\left\|C_{i} \widehat{x}_{i}\right\|\left\|x_{i}\right\| \varphi_{i}\left(y_{i}\right) \\
& \leq \frac{1}{2} \Gamma c_{i 2}\left\|C_{i} \hat{x}_{i}\right\|^{2} \varphi_{i}^{2}\left(y_{i}\right)+\frac{c_{i 2}}{2 \Gamma}\left\|x_{i}\right\|^{2}, \\
& c_{i 3} \rho_{i 2}\left\|C_{i} \widehat{x}_{i}\right\|\left\|x_{i}\right\| \psi_{i}\left(y_{i}\right) \\
& \leq \frac{1}{2} \Gamma c_{i 3} \rho_{i 2}\left\|C_{i} \widehat{x}_{i}\right\|^{2} \psi_{i}^{2}\left(y_{i}\right)+\frac{c_{i 3} \rho_{i 2}}{2 \Gamma}\left\|x_{i}\right\|^{2}, \\
& \bar{c}_{i 6}\left\|C_{i} \widehat{x}_{i}\right\| \sum_{j=1}^{N}\left\|x_{j}\right\|=\bar{c}_{i 6}\left\|C_{i} \widehat{x}_{i}\right\|\left(\left\|x_{1}\right\|+\cdots+\left\|x_{N}\right\|\right) \\
& \leq \frac{1}{2} N \Gamma \bar{c}_{i 6}\left\|C_{i} \widehat{x}_{i}\right\|^{2}+\frac{\bar{c}_{i 6}}{2 \Gamma} \sum_{j=1}^{N}\left\|x_{j}\right\|^{2}, \\
& 2\left\|\bar{e}_{i}\right\|\left\|L_{i}\right\|\left\|x_{i}\right\| \leq \Gamma\left\|L_{i}\right\|^{2}\left\|\bar{e}_{i}\right\|^{2}+\frac{1}{\Gamma}\left\|x_{i}\right\|^{2}, \\
& 2 c_{i 2}\left\|\bar{e}_{i}\right\|\left\|x_{i}\right\| \varphi_{i}\left(y_{i}\right) \leq \Gamma c_{i 2}\left\|\bar{e}_{i}\right\|^{2} \varphi_{i}^{2}\left(y_{i}\right)+\frac{c_{i 2}}{\Gamma}\left\|x_{i}\right\|^{2} \text {, } \\
& 2 c_{i 3} \rho_{i 2}\left\|\bar{e}_{i}\right\|\left\|x_{i}\right\| \psi_{i}\left(y_{i}\right) \\
& \leq \Gamma c_{i 3} \rho_{i 2}\left\|\bar{e}_{i}\right\|^{2} \psi_{i}^{2}\left(y_{i}\right)+\frac{c_{i 3} \rho_{i 2}}{\Gamma}\left\|x_{i}\right\|^{2}, \\
& 2 \bar{c}_{i 6}\left\|\bar{e}_{i}\right\| \sum_{j=1}^{N}\left\|x_{j}\right\|=2 \bar{c}_{i 6}\left\|\bar{e}_{i}\right\|\left(\left\|x_{1}\right\|+\cdots+\left\|x_{N}\right\|\right) \\
& \leq N \Gamma \bar{c}_{i 6}\left\|\bar{e}_{i}\right\|^{2}+\frac{\bar{c}_{i 6}}{\Gamma} \sum_{j=1}^{N}\left\|x_{j}\right\|^{2} .
\end{aligned}
$$

Substituting (21) into (20) gives

$$
\begin{aligned}
\dot{V} & \leq \sum_{i=1}^{N}\left\{-\frac{1}{2} x_{i}^{T} Q_{i} x_{i}-\frac{1}{2} e_{i}^{T} Q_{i} e_{i}+\left(\frac{3}{2 \Gamma}+\frac{3 c_{i 2}}{2 \Gamma}\right.\right. \\
& \left.+\frac{3 c_{i 3} \rho_{i 2}}{2 \Gamma}\right)\left\|x_{i}\right\|^{2}+\frac{3 \bar{c}_{i 6}}{2 \Gamma} \sum_{j=1}^{N}\left\|x_{j}\right\|^{2}+\left\|C_{i} \widehat{x}_{i}\right\|^{2}
\end{aligned}
$$

$$
\begin{aligned}
& \cdot\left[\frac{1}{2} \Gamma\left\|L_{i}\right\|^{2}+\frac{1}{2} \Gamma c_{i 2} \varphi_{i}^{2}\left(y_{i}\right)+\frac{1}{2} \Gamma c_{i 3} \rho_{i 2} \psi_{i}^{2}\left(y_{i}\right)\right. \\
& \left.+\frac{1}{2} N \Gamma \bar{c}_{i 6}\right]+\bar{c}_{i 1}\left\|C_{i} \hat{x}_{i}\right\|+c_{i 3} \rho_{i 1}\left\|C_{i} \hat{x}_{i}\right\| \psi_{i}\left(y_{i}\right) \\
& +c_{i 4}\left\|C_{i} \hat{x}_{i}\right\| c_{i}\left(y_{i}\right)+\left\|\bar{e}_{i}\right\|^{2}\left[\Gamma\left\|L_{i}\right\|^{2}+\Gamma c_{i 2} \varphi_{i}^{2}\left(y_{i}\right)\right. \\
& \left.+\Gamma c_{i 3} \rho_{i 2} \psi_{i}^{2}\left(y_{i}\right)+N \Gamma \bar{c}_{i 6}\right]+2 \bar{c}_{i 1}\left\|\bar{e}_{i}\right\|+2 c_{i 3} \rho_{i 1}\left\|\bar{e}_{i}\right\| \\
& \cdot \psi_{i}\left(y_{i}\right)+2 c_{i 4}\left\|\bar{e}_{i}\right\| c_{i}\left(y_{i}\right)+2\left\|\bar{e}_{i}\right\|\left\|u_{i}\right\| \\
& -\beta_{i 1}^{*}\left\|C_{i} \hat{x}_{i}\right\|^{2} \beta_{m i 1}-\beta_{i 2}^{*}\left\|\bar{e}_{i}\right\|^{2} \beta_{m i 2}-\left(\beta_{i 1}-\beta_{i 1}^{*}\right)^{2} \\
& \left.-\left(\beta_{i 2}-\beta_{i 2}^{*}\right)^{2}\right\} .
\end{aligned}
$$

Since $\sum_{i=1}^{N}\left(\left(3 \bar{c}_{i 6} / 2 \Gamma\right) \sum_{j=1}^{N}\left\|x_{j}\right\|^{2}\right)=\sum_{i=1}^{N}\left(\sum_{j=1}^{N}\left(3 \bar{c}_{j 6} / 2 \Gamma\right)\left\|x_{i}\right\|^{2}\right)$, choosing $\Gamma \geq\left(4 / \lambda_{\min }\left(Q_{i}\right)\right)\left(3 / 2+3 c_{i 2} / 2+3 c_{i 3} \rho_{i 2} / 2+\right.$ $\left.\sum_{j=1}^{N}\left(3 \bar{c}_{j 6} / 2\right)\right)$, for example, $(1 / 4) \lambda_{\min }\left(Q_{i}\right) \geq\left(3 / 2 \Gamma+3 c_{i 2} / 2 \Gamma+\right.$ $\left.3 c_{i 3} \rho_{i 2} / 2 \Gamma+\sum_{j=1}^{N}\left(3 \bar{c}_{j 6} / 2 \Gamma\right)\right)$, then

$$
\begin{aligned}
\dot{V} & \leq \sum_{i=1}^{N}\left\{-\frac{1}{4} x_{i}^{T} Q_{i} x_{i}-\frac{1}{4} e_{i}^{T} Q_{i} e_{i}+\bar{c}_{i 1}\left\|C_{i} \widehat{x}_{i}\right\|\right. \\
& +c_{i 3} \rho_{i 1}\left\|C_{i} \widehat{x}_{i}\right\| \psi_{i}\left(y_{i}\right)+c_{i 4}\left\|C_{i} \widehat{x}_{i}\right\| c_{i}\left(y_{i}\right)+\left\|C_{i} \widehat{x}_{i}\right\|^{2} \\
& +\left[\frac{1}{2} \Gamma\left\|L_{i}\right\|^{2}+\frac{1}{2} \Gamma c_{i 2} \varphi_{i}^{2}\left(y_{i}\right)+\frac{1}{2} \Gamma c_{i 3} \rho_{i 2} \psi_{i}^{2}\left(y_{i}\right)\right. \\
& \left.+\frac{1}{2} N \Gamma \bar{c}_{i 6}\right]+2 \bar{c}_{i 1}\left\|\bar{e}_{i}\right\|+2 c_{i 3} \rho_{i 1}\left\|\bar{e}_{i}\right\| \psi_{i}\left(y_{i}\right) \\
& +2 c_{i 4}\left\|\bar{e}_{i}\right\| \varsigma_{i}\left(y_{i}\right)+2\left\|\bar{e}_{i}\right\|\left\|u_{i}\right\|+\left\|\bar{e}_{i}\right\|^{2}\left[\Gamma\left\|L_{i}\right\|^{2}\right. \\
& \left.+\Gamma c_{i 2} \varphi_{i}^{2}\left(y_{i}\right)+\Gamma c_{i 3} \rho_{i 2} \psi_{i}^{2}\left(y_{i}\right)+N \Gamma \bar{c}_{i 6}\right] \\
& -\beta_{i 1}^{*}\left\|C_{i} \hat{x}_{i}\right\|^{2} \beta_{m i 1}-\beta_{i 2}^{*}\left\|\bar{e}_{i}\right\|^{2} \beta_{m i 2}-\left(\beta_{i 1}-\beta_{i 1}^{*}\right)^{2} \\
& \left.-\left(\beta_{i 2}-\beta_{i 2}^{*}\right)^{2}\right\} .
\end{aligned}
$$

Substituting (5), (7), and (10) into (23) gives

$$
\begin{aligned}
\dot{V} & \leq \sum_{i=1}^{N}\left\{-\frac{1}{4} x_{i}^{T} Q_{i} x_{i}-\frac{1}{4} e_{i}^{T} Q_{i} e_{i}+\bar{c}_{i 1}\left\|C_{i} \hat{x}_{i}\right\|\right. \\
& +c_{i 3} \rho_{i 1}\left\|C_{i} \widehat{x}_{i}\right\| \psi_{i}\left(y_{i}\right)+c_{i 4}\left\|C_{i} \widehat{x}_{i}\right\| c_{i}\left(y_{i}\right)+\left\|C_{i} \widehat{x}_{i}\right\|^{2} \\
& \cdot\left[\frac{1}{2} \Gamma\left\|L_{i}\right\|^{2}+\frac{1}{2} \Gamma c_{i 2} \varphi_{i}^{2}\left(y_{i}\right)+\frac{1}{2} \Gamma c_{i 3} \rho_{i 2} \psi_{i}^{2}\left(y_{i}\right)\right. \\
& \left.+\frac{1}{2} N \Gamma \bar{c}_{i 6}\right]+2 \bar{c}_{i 1}\left\|\bar{e}_{i}\right\|+2 c_{i 3} \rho_{i 1}\left\|\bar{e}_{i}\right\| \psi_{i}\left(y_{i}\right) \\
& +2 c_{i 4}\left\|\bar{e}_{i}\right\| \varsigma_{i}\left(y_{i}\right)+2\left\|\bar{e}_{i}\right\|\left\|\beta_{i 1} C_{i} \hat{x}_{i} \beta_{m i 1}\right\|+\left\|\bar{e}_{i}\right\|^{2} \\
& \cdot\left[\Gamma\left\|L_{i}\right\|^{2}+\Gamma c_{i 2} \varphi_{i}^{2}\left(y_{i}\right)+\Gamma c_{i 3} \rho_{i 2} \psi_{i}^{2}\left(y_{i}\right)+N \Gamma \bar{c}_{i 6}\right]
\end{aligned}
$$




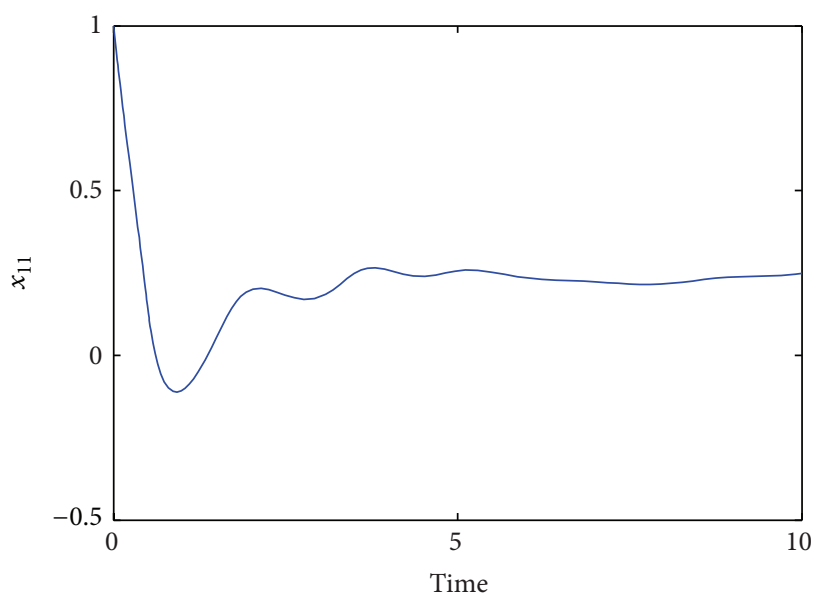

(a)

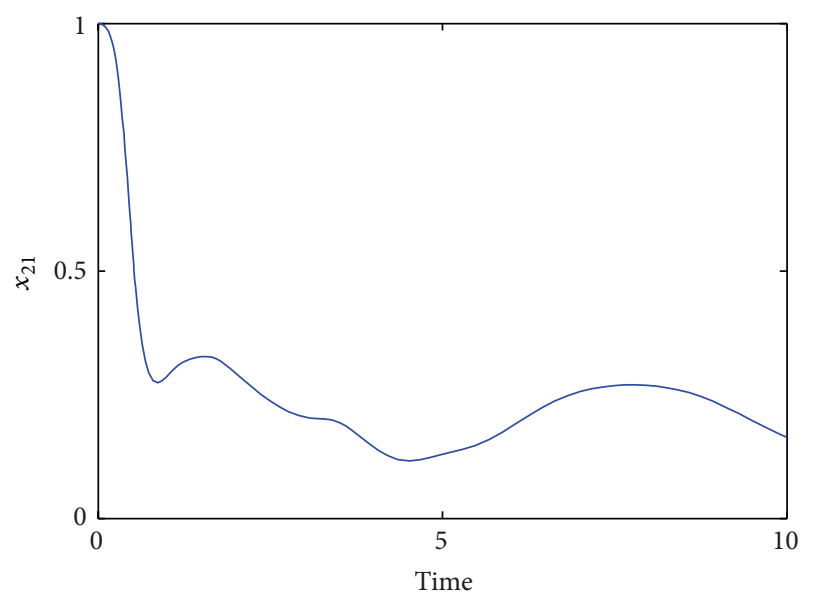

(c)

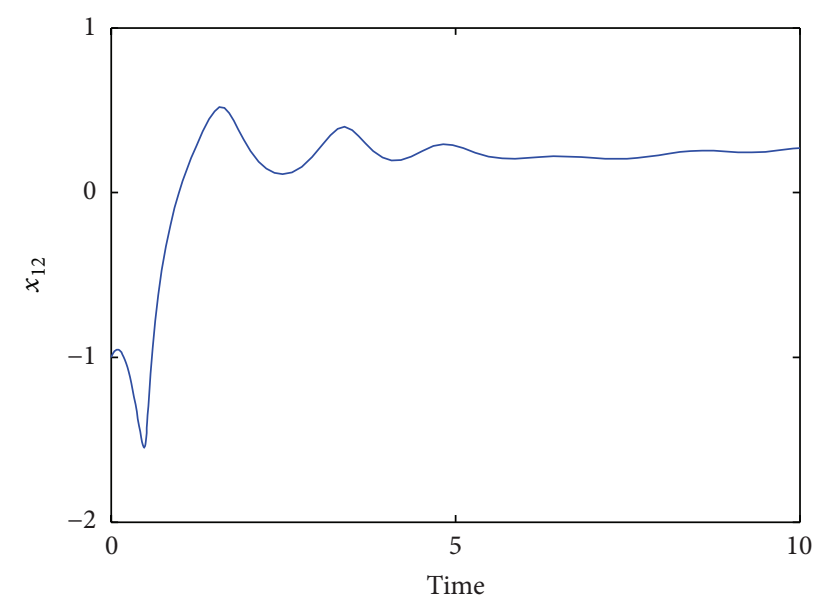

(b)

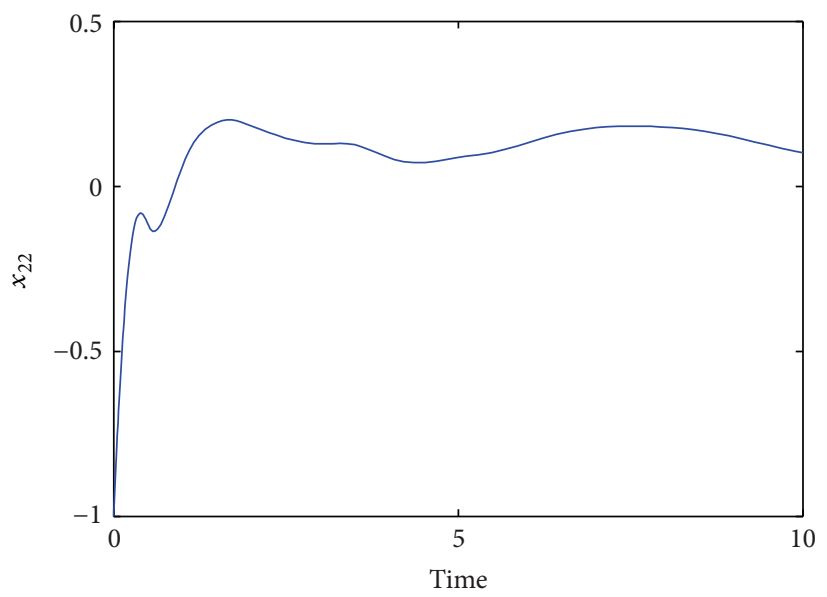

(d)

FIGURE 1: Simulation results of the system states with $\beta_{11}^{*}=0.8, \beta_{12}^{*}=1, \beta_{21}^{*}=1.2$, and $\beta_{22}^{*}=1.4$.

$$
\begin{aligned}
& -\beta_{i 1}^{*}\left\|C_{i} \widehat{x}_{i}\right\|^{2}\left[\frac{1}{2}+\psi_{i}^{2}\left(y_{i}\right)+\varsigma_{i}^{2}\left(y_{i}\right)\right. \\
& \left.+\left(\frac{1}{2}+\varphi_{i}^{2}\left(y_{i}\right)+\psi_{i}^{2}\left(y_{i}\right)\right)\right]-\beta_{i 2}^{*}\left\|\bar{e}_{i}\right\|^{2}\left[\frac{1}{2}\right. \\
& +\psi_{i}^{2}\left(y_{i}\right)+\varsigma_{i}^{2}\left(y_{i}\right)+\left\|\beta_{i 1} C_{i} \widehat{x}_{i} \beta_{m i 1}\right\|^{2} \\
& \left.+\left(\frac{1}{2}+\varphi_{i}^{2}\left(y_{i}\right)+\psi_{i}^{2}\left(y_{i}\right)\right)\right]-\left(\beta_{i 1}-\beta_{i 1}^{*}\right)^{2}-\left(\beta_{i 2}\right. \\
& \left.\left.-\beta_{i 2}^{*}\right)^{2}\right\} .
\end{aligned}
$$

Choosing $\beta_{i 1}^{*} \geq \min \left(\Gamma\left\|L_{i}\right\|^{2}+N \Gamma \bar{c}_{i 6},(1 / 2) \Gamma c_{i 2},(1 / 2) \Gamma c_{i 3} \rho_{i 2}\right)$, $\beta_{i 2}^{*} \geq \min \left(2 \Gamma\left\|L_{i}\right\|^{2}+2 N \Gamma \bar{c}_{i 6}, \Gamma c_{i 2}, \Gamma c_{i 3} \rho_{i 2}\right)$ and completing the squares yield

$$
\begin{aligned}
\dot{V} & \leq \sum_{i=1}^{N}\left\{-\frac{1}{4} x_{i}^{T} Q_{i} x_{i}-\frac{1}{4} e_{i}^{T} Q_{i} e_{i}\right. \\
& -\frac{1}{2} \beta_{i 1}^{*}\left[\left\|C_{i} \widehat{x}_{i}\right\|-\frac{\bar{c}_{i 1}}{\beta_{i 1}^{*}}\right]^{2}+\frac{\bar{c}_{i 1}^{2}}{2 \beta_{i 1}^{*}}
\end{aligned}
$$

$$
\begin{aligned}
& -\beta_{i 1}^{*}\left[\left\|C_{i} \hat{x}_{i}\right\| \psi_{i}\left(y_{i}\right)-\frac{c_{i 3} \rho_{i 1}}{2 \beta_{i 1}^{*}}\right]^{2}+\frac{\left(c_{i 3} \rho_{i 1}\right)^{2}}{4 \beta_{i 1}^{*}} \\
& -\beta_{i 1}^{*}\left[\left\|C_{i} \widehat{x}_{i}\right\| c_{i}\left(y_{i}\right)-\frac{c_{i 4}}{2 \beta_{i 1}^{*}}\right]^{2}+\frac{c_{i 4}^{2}}{4 \beta_{i 1}^{*}} \\
& -\frac{1}{2} \beta_{i 2}^{*}\left[\left\|\bar{e}_{i}\right\|-\frac{2 \bar{c}_{i 1}}{\beta_{i 2}^{*}}\right]^{2}+\frac{2 \bar{c}_{i 1}^{2}}{\beta_{i 2}^{*}} \\
& -\beta_{i 2}^{*}\left[\left\|\bar{e}_{i}\right\| \psi_{i}\left(y_{i}\right)-\frac{c_{i 3} \rho_{i 1}}{\beta_{i 2}^{*}}\right]^{2}+\frac{\left(c_{i 3} \rho_{i 1}\right)^{2}}{\beta_{i 2}^{*}} \\
& -\beta_{i 2}^{*}\left[\left\|\bar{e}_{i}\right\| \varsigma_{i}\left(y_{i}\right)-\frac{c_{i 4}}{\beta_{i 2}^{*}}\right]^{2}+\frac{c_{i 4}^{2}}{\beta_{i 2}^{*}} \\
& -\beta_{i 2}^{*}\left[\left\|\bar{e}_{i}\right\|\left\|\beta_{i 1} C_{i} \widehat{x}_{i} \beta_{m i 1}\right\|-\frac{1}{\beta_{i 2}^{*}}\right]^{2}+\frac{1}{\beta_{i 2}^{*}} \\
& -\left(\beta_{i 1}-\beta_{i 1}^{*}\right)^{2}-\left(\beta_{i 2}-\beta_{i 2}^{*}\right)^{2} \cdot
\end{aligned}
$$




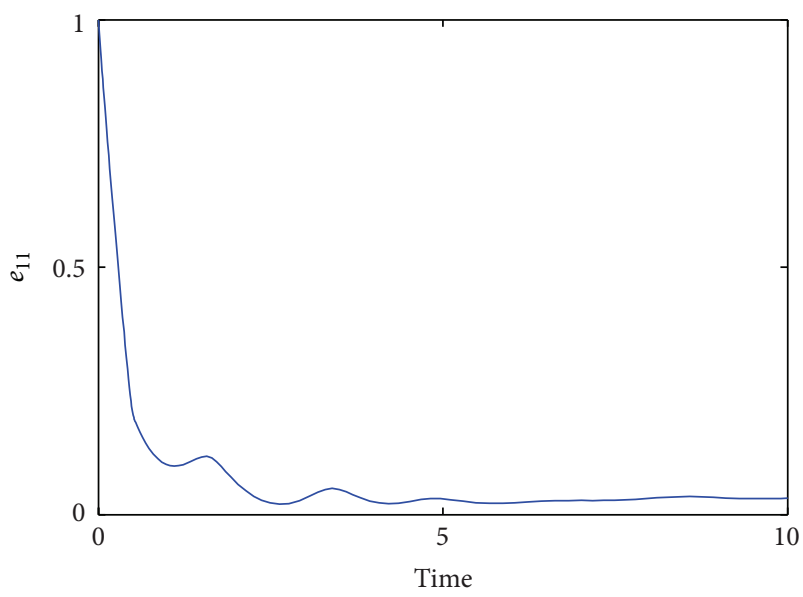

(a)

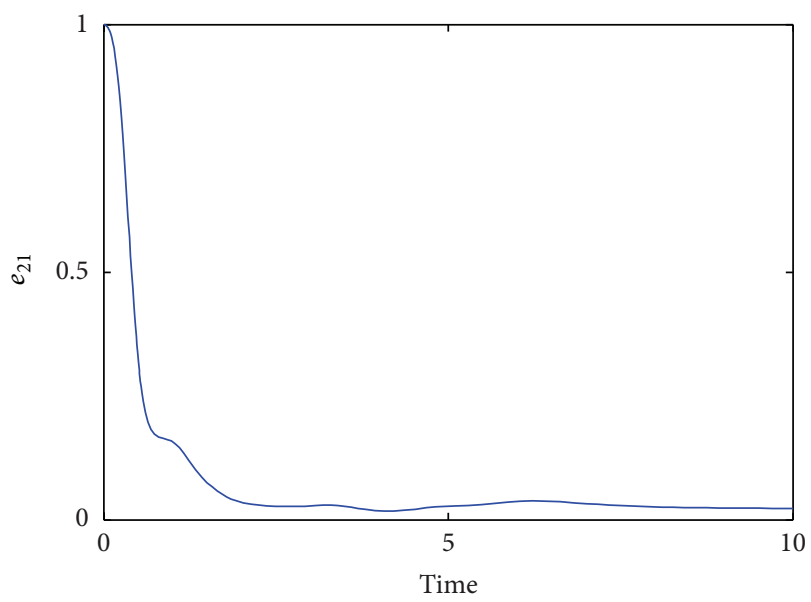

(c)

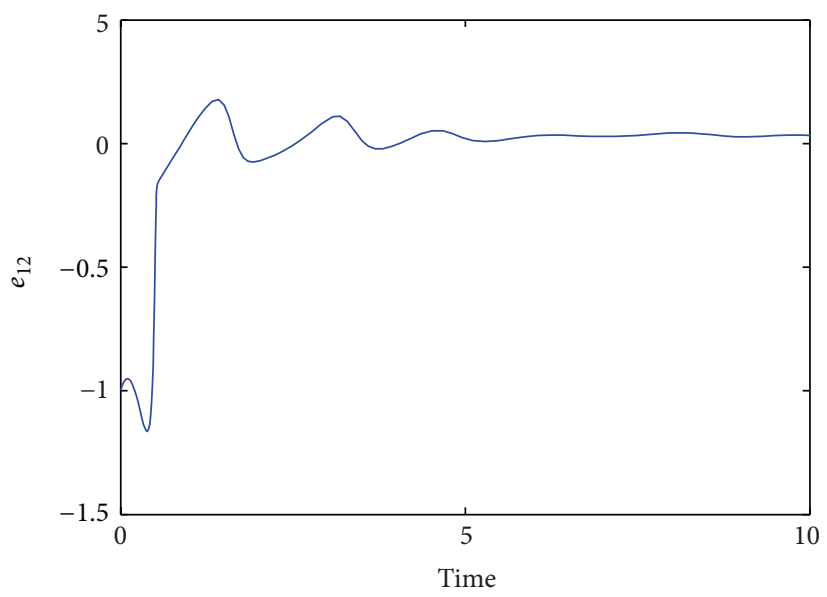

(b)

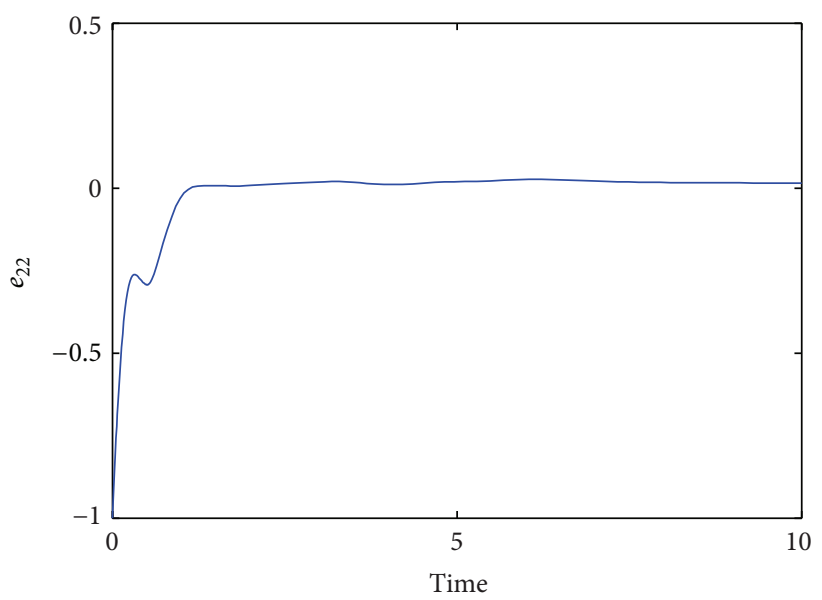

(d)

FIGURE 2: Simulation results of the estimation errors with $\beta_{11}^{*}=0.8, \beta_{12}^{*}=1, \beta_{21}^{*}=1.2$, and $\beta_{22}^{*}=1.4$.

Equation (25) can be written as

$$
\begin{aligned}
\dot{V} & \leq \sum_{i=1}^{N}\left\{-\frac{1}{4} x_{i}^{T} Q_{i} x_{i}-\frac{1}{4} e_{i}^{T} Q_{i} e_{i}-\left(\beta_{i 1}-\beta_{i 1}^{*}\right)^{2}\right. \\
& \left.-\left(\beta_{i 2}-\beta_{i 2}^{*}\right)^{2}+M\right\} \leq-\mu V+M,
\end{aligned}
$$

where

$$
\begin{aligned}
\mu & =\min \left\{\frac{\lambda_{\min }\left(Q_{i}\right)}{2 \lambda_{\max }\left(P_{i}\right)}, 2\right\}, \quad(1 \leq i \leq N), \\
M & =\sum_{i=1}^{N}\left\{\frac{1}{4 \beta_{i 1}^{*}}\left[2 \bar{c}_{i 1}^{2}+\left(c_{i 3} \rho_{i 1}\right)^{2}+c_{i 4}^{2}\right]\right. \\
& \left.+\frac{1}{\beta_{i 2}^{*}}\left[1+2 \bar{c}_{i 1}^{2}+\left(c_{i 3} \rho_{i 1}\right)^{2}+c_{i 4}^{2}\right]\right\} .
\end{aligned}
$$

Therefore, $V\left(x, e, \widetilde{\beta}_{1}, \widetilde{\beta}_{2}\right)$, where vectors $\widetilde{\beta}_{1}=\beta_{i 1}-\beta_{i 1}^{*}$ and $\widetilde{\beta}_{2}=\beta_{i 2}-\beta_{i 2}^{*}, i=1,2, \ldots, N$, decreases mono- tonically until $\left(x, e, \widetilde{\beta}_{1}, \widetilde{\beta}_{2}\right)$ reaches the compact set $\Theta=$ $\left\{V\left(x, e, \widetilde{\beta}_{1}, \widetilde{\beta}_{2}\right) \leq \mu^{-1} M\right\}$. This means that $x, e, \widetilde{\beta}_{1}$, and $\tilde{\beta}_{2}$ are uniformly bounded, and so is $\beta_{i 1}$ and $\beta_{i 2}, i=$ $1,2, \ldots, N$. Furthermore, from (26) to (28), it can be seen that choosing the design parameters $\beta_{i 1}^{*}$ and $\beta_{i 2}^{*}, i=1,2, \ldots, N$, appropriately large, will reduce the residual error bound $\mu^{-1} M$ and the system states $x$ and the estimation errors $e$ will approach arbitrarily small values.

Remark 8. Since the Lyapunov function (12) contains the terms $\left(\beta_{i 1}-\beta_{i 1}^{*}\right)^{2}$ and $\left(\beta_{i 2}-\beta_{i 2}^{*}\right)^{2}, i=1,2, \ldots, N,\left(\beta_{i 1}-\beta_{i 1}^{*}\right)$ and $\left(\beta_{i 2}-\beta_{i 2}^{*}\right)$ are expected to be zero or very small values; that is, $\beta_{i 1}=\beta_{i 1}^{*}$ and $\beta_{i 2}=\beta_{i 2}^{*}$ are desired. Gradually increasing the value of $\beta_{i 1}(t)$ and $\beta_{i 2}(t)$ from small $\beta_{i 1}(0)$ and $\beta_{i 2}(0)$ to $\beta_{i 1}^{*}$ and $\beta_{i 2}^{*}$, which are usually large to get small system states and estimation errors, by adaptive law (6) and (9) is necessary to maintain the stability of the closed system. In each subsystem, if we set $\beta_{i 1}=\beta_{i 1}^{*}$ and $\beta_{i 2}=\beta_{i 2}^{*}$ without using adaptive law (6) and (9), the controller and observer are high gain controller and observer, which will exhibit a 


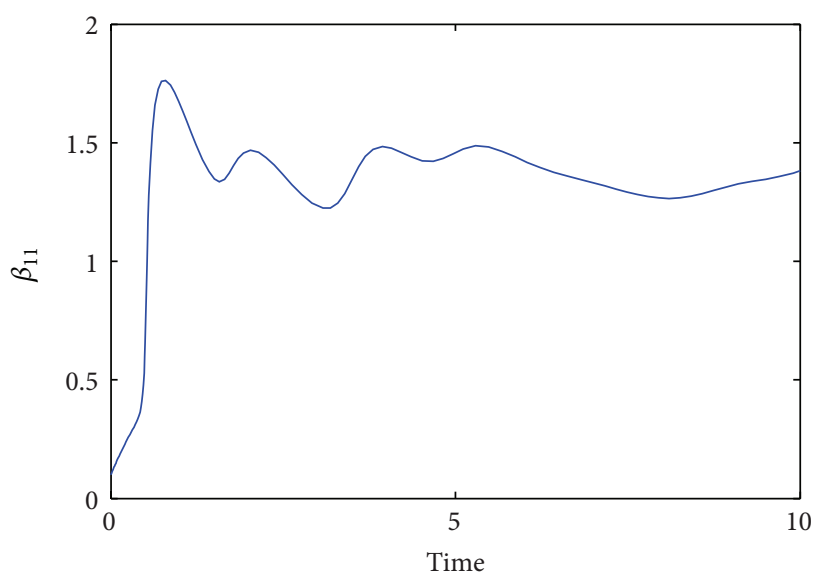

(a)

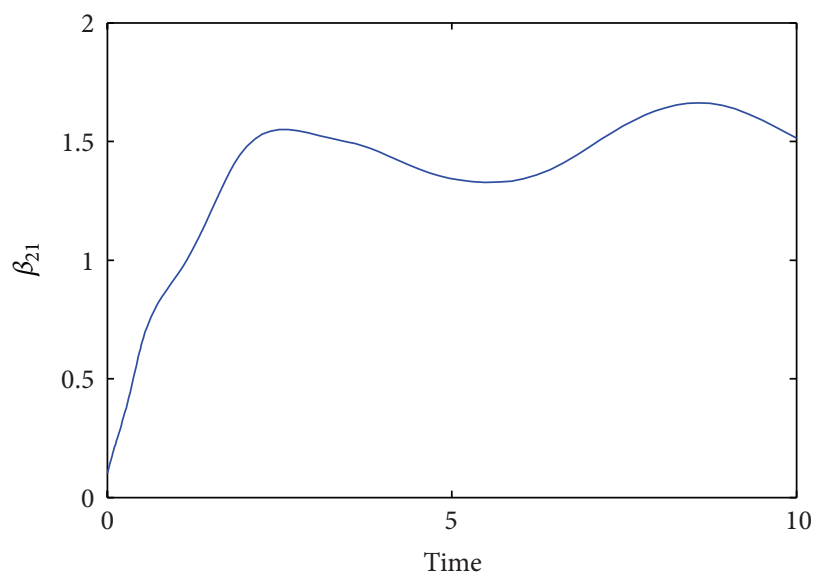

(c)

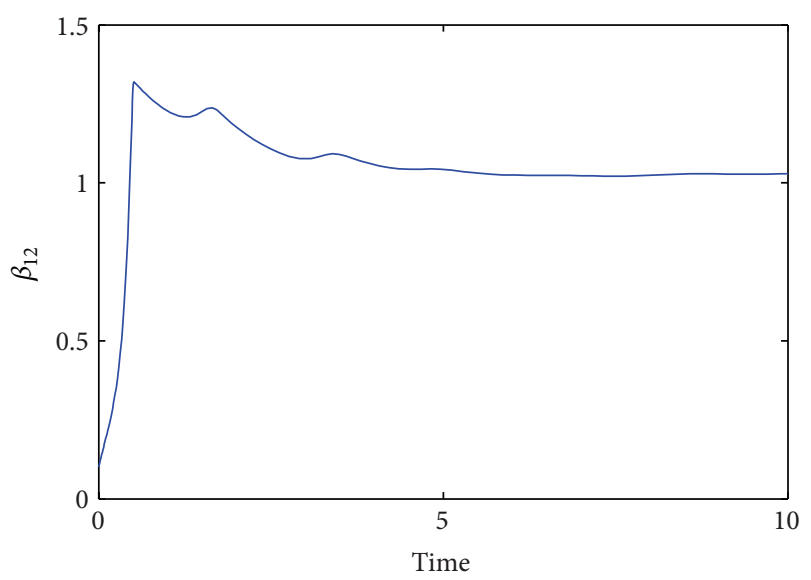

(b)

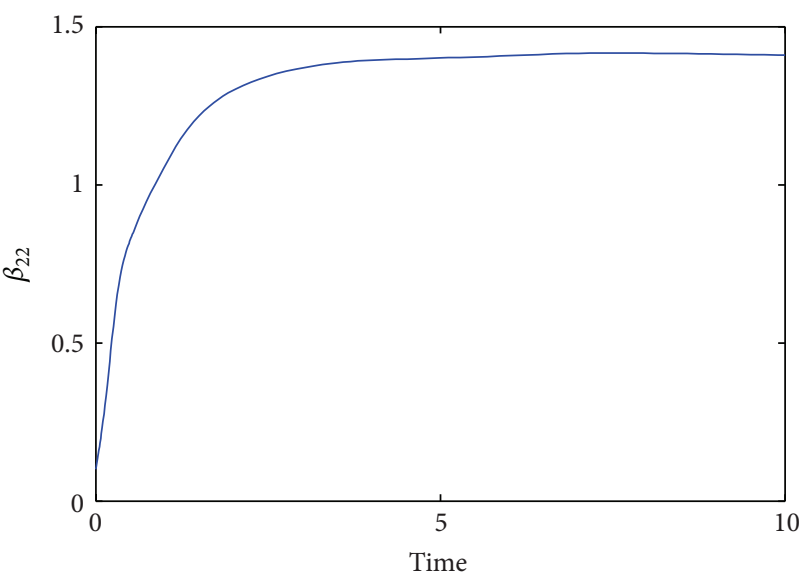

(d)

FIGURE 3: Simulation results of the adaptive parameters with $\beta_{11}^{*}=0.8, \beta_{12}^{*}=1, \beta_{21}^{*}=1.2$, and $\beta_{22}^{*}=1.4$.

peaking phenomenon [21] in the transient behavior due to the fact that the large initial control error and estimation error are multiplied by the high gains $\beta_{i 1}$ and $\beta_{i 2}$, respectively (see (5) and (8)). As indicated in [22], such peaking acts as destabilizing input and may even cause the closed system to be unstable.

To use the proposed robust adaptive observer in practice, we should follow the following steps.

Step 1. Determine $A_{i}, B_{i}$, and $C_{i}$ by the structure of the system model.
Step 2. Determine the nonlinear functions $\varphi_{i}\left(y_{i}\right), \psi_{i}\left(y_{i}\right)$, and $\varsigma_{i}\left(y_{i}\right)$ by experience.

Step 3. Choosing appropriate $L_{i}, \beta_{i 1}^{*}$, and $\beta_{i 2}^{*}$, the decentralized robust adaptive controller given by (5)-(7) and the decentralized robust adaptive observer given by (8) and (9) can be obtained.

\section{Illustration}

Consider an interconnected nonlinear system $S$ consisting of two subsystems

$$
\begin{aligned}
& S_{1}:\left\{\begin{array}{l}
{\left[\begin{array}{l}
\dot{x}_{11} \\
\dot{x}_{12}
\end{array}\right]=\left[\begin{array}{cc}
-1 & 1 \\
1 & 2
\end{array}\right]\left[\begin{array}{l}
x_{11} \\
x_{12}
\end{array}\right]+\left[\begin{array}{l}
0 \\
1
\end{array}\right]\left[u_{1}+f_{1}\left(x_{1}, \omega_{1}, \theta_{1}, d_{1}\right)+H_{12}\left(x_{2}, y_{2}, \omega_{2}, \theta_{2}\right)\right]} \\
S_{2}:\left\{\begin{array}{l}
\dot{x}_{21} \\
\dot{x}_{22} \\
y_{2}=x_{21}+x_{22},
\end{array}\right]=\left[\begin{array}{cc}
-1 & 1 \\
1 & -2
\end{array}\right]\left[\begin{array}{l}
x_{21} \\
x_{22}
\end{array}\right]+\left[\begin{array}{l}
1 \\
1
\end{array}\right]\left[u_{2}+f_{2}\left(x_{2}, \omega_{2}, \theta_{2}, d_{2}\right)+H_{21}\left(x_{1}, y_{1}, \omega_{1}, \theta_{1}\right)\right]
\end{array}\right.
\end{aligned}
$$




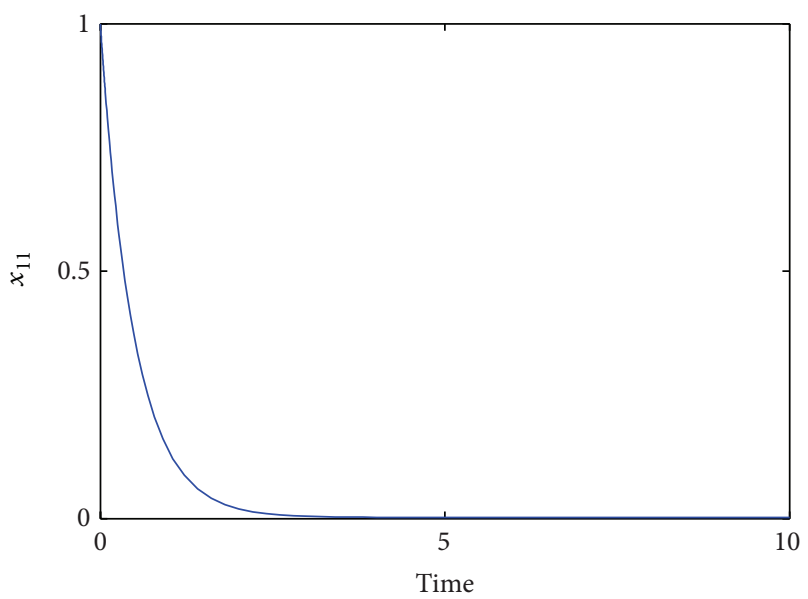

(a)

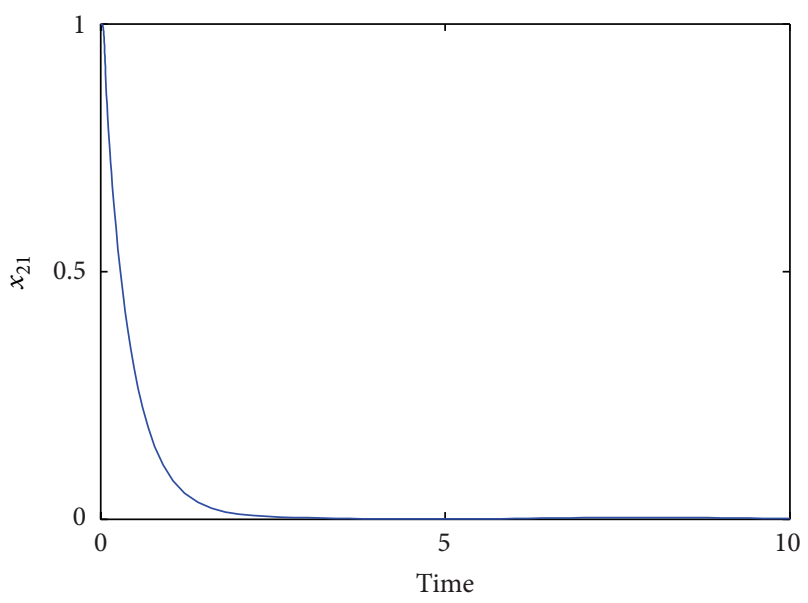

(c)

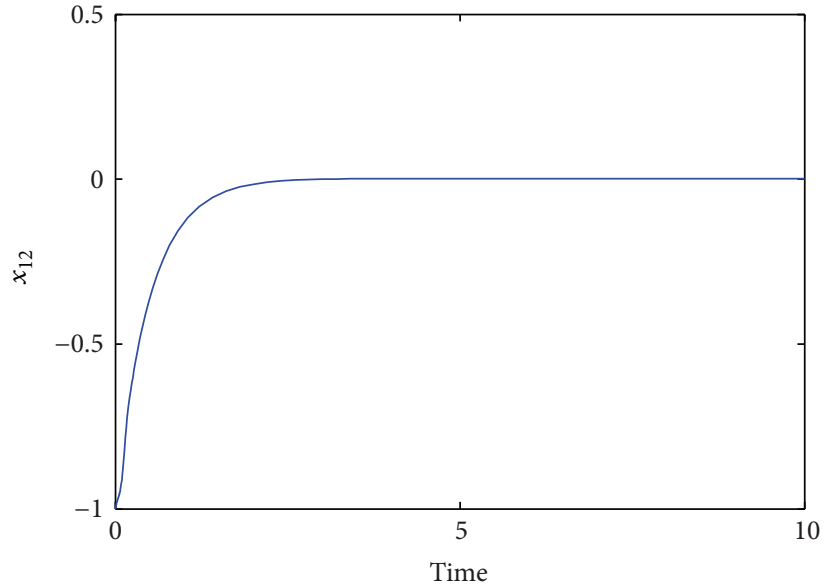

(b)

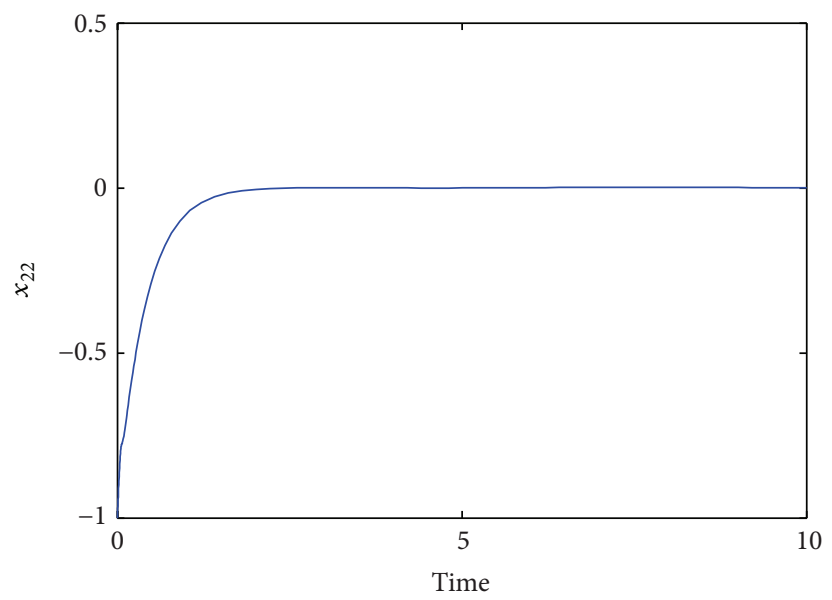

(d)

FIGURE 4: Simulation results of the system states with $\beta_{11}^{*}=80, \beta_{12}^{*}=100, \beta_{21}^{*}=120$, and $\beta_{22}^{*}=140$.

where $f_{1}(\cdot), f_{2}(\cdot), H_{12}(\cdot)$, and $H_{21}(\cdot)$ are unknown nonlinear functions and have the following forms:

$$
\begin{aligned}
f_{1}\left(x_{1}, \omega_{1}, \theta_{1}, d_{1}\right)= & \theta_{11} x_{11}\left(x_{11}+x_{12}\right)^{2}+\theta_{12} \omega_{1} \\
& +\theta_{13}\left(x_{11}+x_{12}\right)+d_{1}, \\
f_{2}\left(x_{2}, \omega_{2}, \theta_{2}, d_{2}\right)= & \theta_{21} x_{22}\left(x_{21}+x_{22}\right)^{2}+\theta_{22} \omega_{2} \\
& +\theta_{23}\left(x_{21}+x_{22}\right)+d_{2}, \\
H_{12}\left(x_{2}, y_{2}, \omega_{2}, \theta_{2}\right)= & \theta_{21} x_{22}+\theta_{22}\left(x_{21}+x_{22}\right) \\
& +\theta_{23} \omega_{2}, \\
H_{21}\left(x_{1}, y_{1}, \omega_{1}, \theta_{1}\right)= & \theta_{11} x_{11}+\theta_{12} \omega_{1} \\
& +\theta_{13}\left(x_{11}+x_{12}\right),
\end{aligned}
$$

where $\theta_{11}, \theta_{12}, \theta_{13}, \theta_{21}, \theta_{22}$, and $\theta_{23}$ are unknown parameters; $d_{1}$ and $d_{2}$ are bounded disturbances; $\omega_{1}$ and $\omega_{2}$ are unmodeled dynamics described by

$$
\begin{aligned}
& \dot{\omega}_{1}=-\omega_{1}+\sin \left(x_{12}\right), \\
& \dot{\omega}_{2}=-\omega_{2}+x_{22} .
\end{aligned}
$$

From (30), we have

$$
\begin{aligned}
\left\|f_{1}\left(x_{1}, \omega_{1}, \theta_{1}, d_{1}\right)\right\| \leq & c_{11}+c_{12}\left\|x_{1}\right\| y_{1}^{2}+c_{13}\left\|\omega_{1}\right\| \\
& +c_{14}\left|y_{1}\right|, \\
\left\|f_{2}\left(x_{2}, \omega_{2}, \theta_{2}, d_{2}\right)\right\| \leq & c_{21}+c_{22}\left\|x_{2}\right\| y_{2}^{2}+c_{23}\left\|\omega_{2}\right\| \\
& +c_{24}\left|y_{2}\right|, \\
\left\|H_{12}\left(x_{2}, y_{2}, \omega_{2}, \theta_{2}\right)\right\| \leq & c_{15}+c_{16}\left\|x_{2}\right\|+c_{17}\left\|y_{2}\right\| \\
& +c_{18}\left\|\omega_{2}\right\|, \\
\left\|H_{21}\left(x_{1}, y_{1}, \omega_{1}, \theta_{1}\right)\right\|= & c_{25}+c_{26}\left\|x_{1}\right\|+c_{27}\left\|y_{1}\right\| \\
& +c_{28}\left\|\omega_{1}\right\|,
\end{aligned}
$$

where $c_{i j} \geq 0, i=1,2$ and $j=1,2, \ldots, 8$. Comparing (32) with (3) in Assumption 3, we have $\varphi_{1}\left(y_{1}\right)=y_{1}^{2}, \psi_{1}\left(y_{1}\right)=$ $1, \varsigma_{1}\left(y_{1}\right)=\left|y_{1}\right|, \varphi_{2}\left(y_{2}\right)=y_{2}^{2}, \psi_{2}\left(y_{2}\right)=1$, and $\varsigma_{2}\left(y_{2}\right)=\left|y_{2}\right|$. 


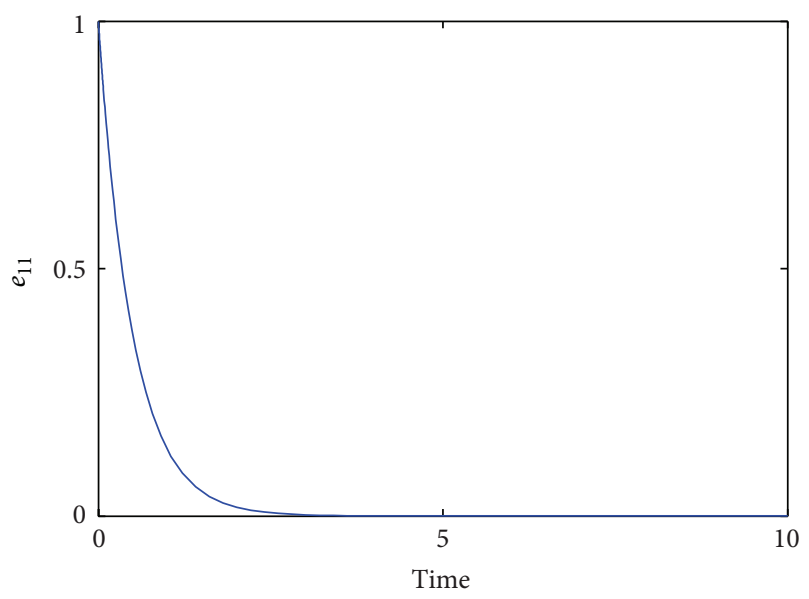

(a)

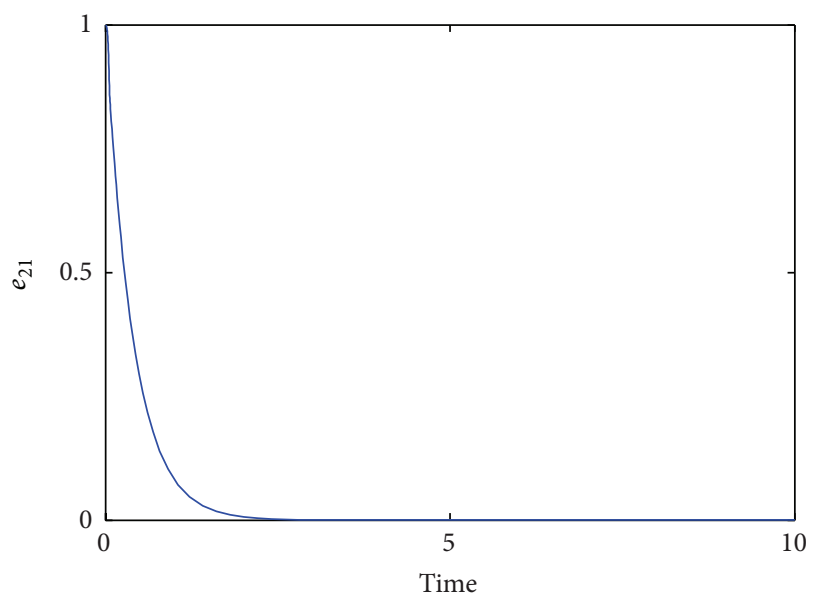

(c)

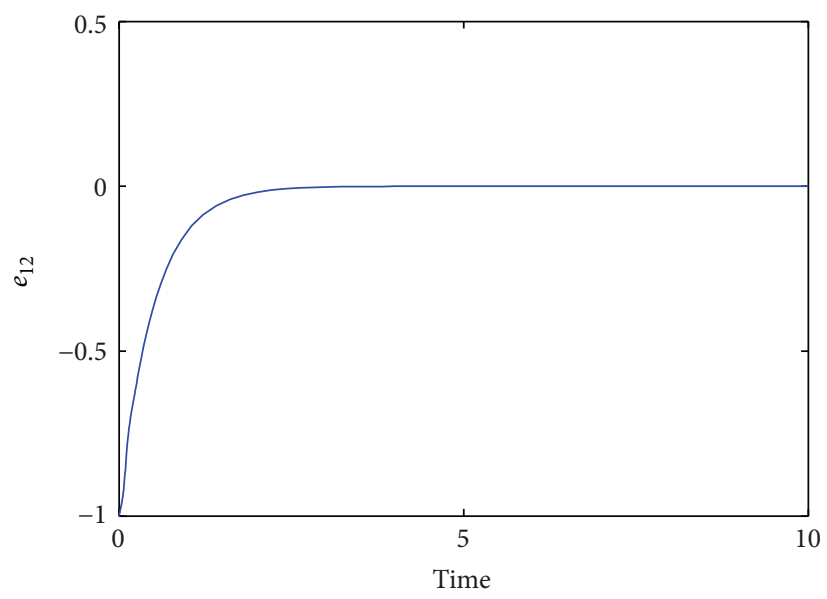

(b)

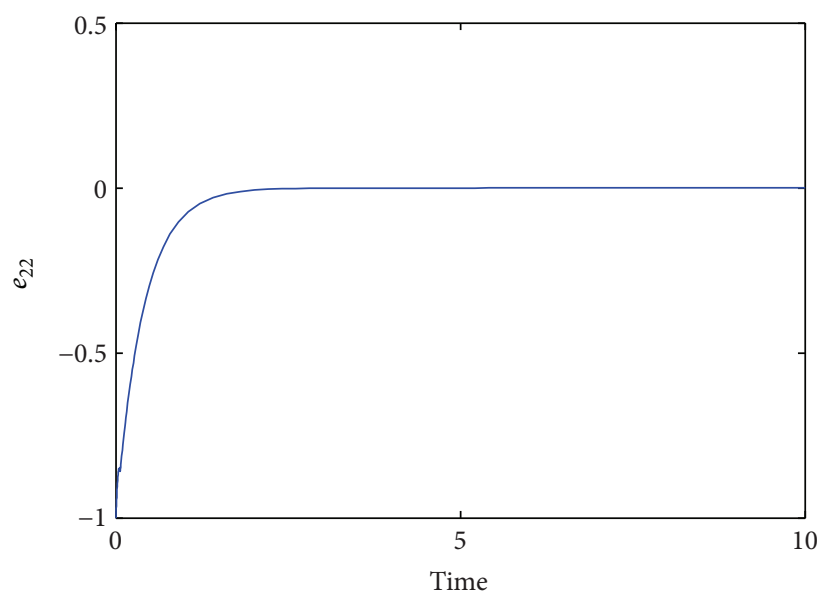

(d)

FIGURE 5: Simulation results of the estimation errors with $\beta_{11}^{*}=80, \beta_{12}^{*}=100, \beta_{21}^{*}=120$, and $\beta_{22}^{*}=140$.

Taking $L_{1}=\left[\begin{array}{ll}1 & 4\end{array}\right]$ and $L_{2}=\left[\begin{array}{ll}1 & 1\end{array}\right]$, we can check that Assumption 6 is satisfied with

$$
\begin{aligned}
P_{1} & =\left[\begin{array}{ll}
2 & 1 \\
1 & 1
\end{array}\right], \\
P_{2} & =\left[\begin{array}{cc}
2 & -1 \\
-1 & 2
\end{array}\right], \\
Q_{1} & =\left[\begin{array}{ll}
4 & 1 \\
1 & 2
\end{array}\right], \\
Q_{2} & =\left[\begin{array}{cc}
8 & -5 \\
-5 & 12
\end{array}\right] .
\end{aligned}
$$

From (5)-(10), the decentralized robust adaptive output feedback stabilization for subsystem (29) is as follows.

For subsystem $S_{1}$, consider the following.
Decentralized robust adaptive controller is

$$
\begin{aligned}
u_{1} & =-\beta_{11}\left(\widehat{x}_{11}+\widehat{x}_{12}\right) \beta_{m 11}, \\
\dot{\beta}_{11} & =\left\|\widehat{x}_{11}+\widehat{x}_{12}\right\|^{2} \beta_{m 11}-\beta_{11}+\beta_{11}^{*}, \\
\beta_{m 11} & =3+y_{1}^{2}+y_{1}^{4} .
\end{aligned}
$$

Decentralized robust adaptive observer is

$$
\begin{aligned}
{\left[\begin{array}{l}
\dot{\hat{x}}_{11} \\
\dot{\hat{x}}_{12}
\end{array}\right]=} & {\left[\begin{array}{cc}
-1 & 1 \\
0 & -2
\end{array}\right]\left[\begin{array}{l}
\hat{x}_{11} \\
\hat{x}_{12}
\end{array}\right] } \\
& +\beta_{12}\left[\begin{array}{l}
0 \\
1
\end{array}\right]\left(y_{1}-\widehat{x}_{11}-\widehat{x}_{12}\right) \beta_{m 12}, \\
\dot{\beta}_{12}= & \left\|y_{1}-\hat{x}_{11}-\hat{x}_{12}\right\|^{2} \beta_{m 12}-\beta_{12}+\beta_{12}^{*}, \\
\beta_{m 12}= & 3+y_{1}^{2}+y_{1}^{4}+\left\|\beta_{11}\left(\hat{x}_{11}+\hat{x}_{12}\right) \beta_{m 11}\right\|^{2} .
\end{aligned}
$$

For subsystem $S_{2}$, consider the following. 


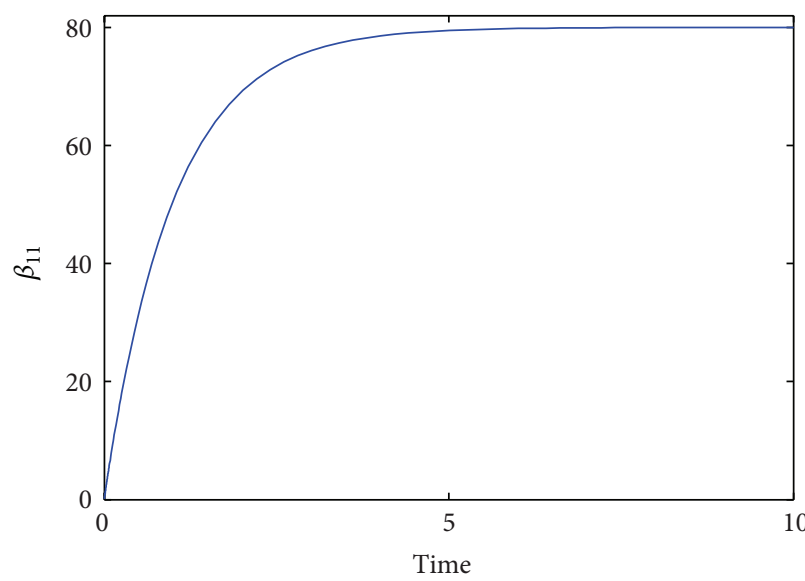

(a)

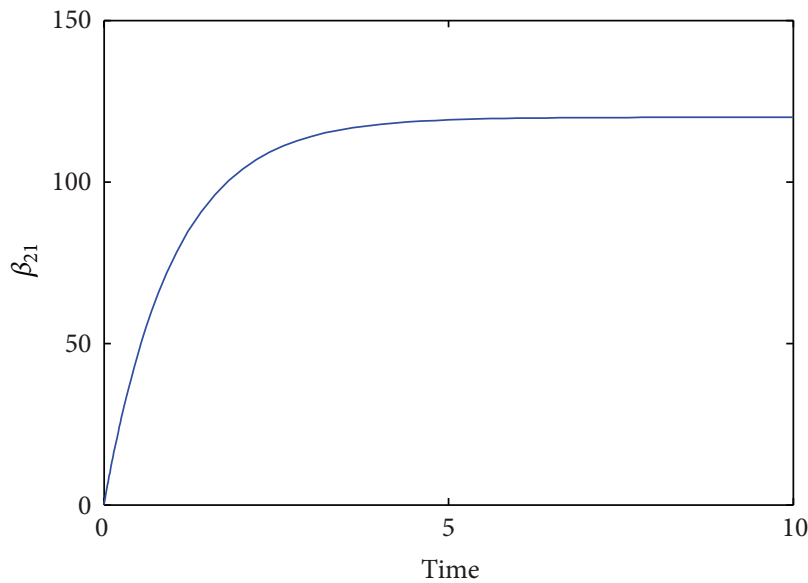

(c)

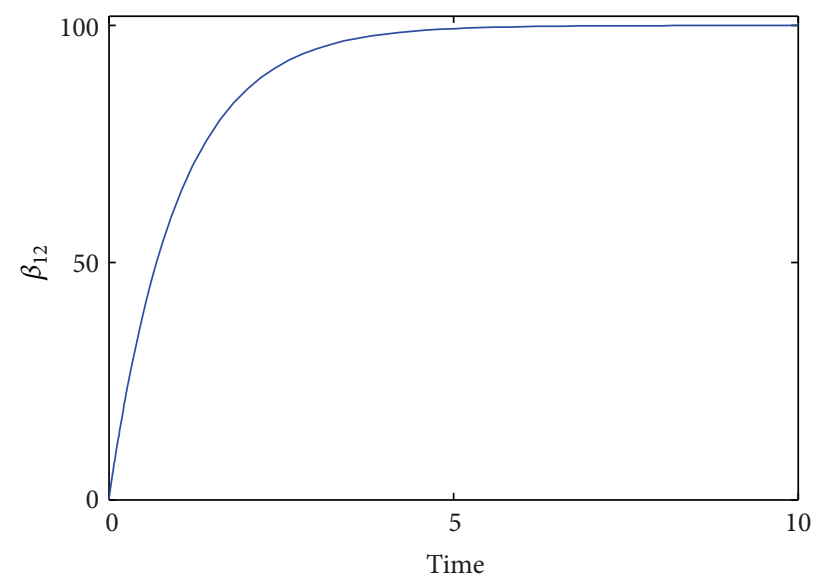

(b)

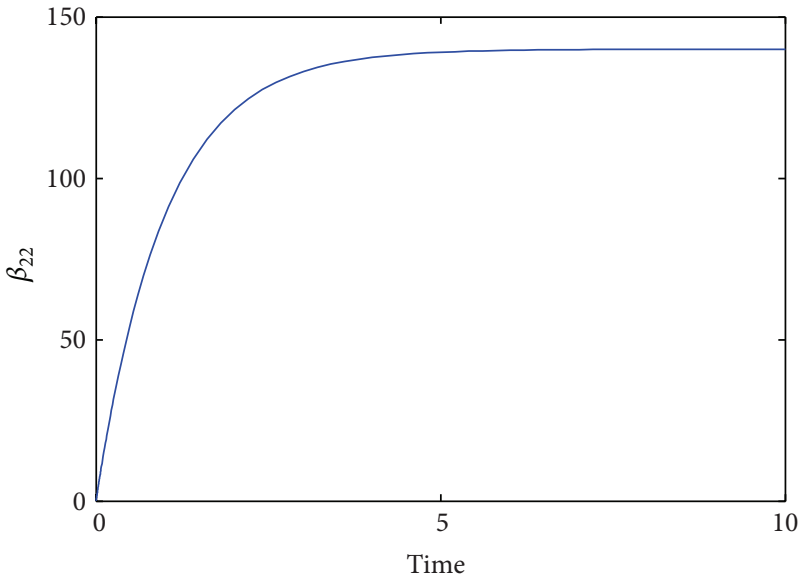

(d)

FIGURE 6: Simulation results of the adaptive parameters with $\beta_{11}^{*}=80, \beta_{12}^{*}=100, \beta_{21}^{*}=120$, and $\beta_{22}^{*}=140$.

Decentralized robust adaptive controller is

$$
\begin{aligned}
u_{2} & =-\beta_{21}\left(\hat{x}_{21}+\hat{x}_{22}\right) \beta_{m 21}, \\
\dot{\beta}_{21} & =\left\|\widehat{x}_{21}+\hat{x}_{22}\right\|^{2} \beta_{m 21}-\beta_{21}+\beta_{21}^{*}, \\
\beta_{m 21} & =3+y_{2}^{2}+y_{2}^{4} .
\end{aligned}
$$

Decentralized robust adaptive observer is

$$
\begin{aligned}
{\left[\begin{array}{c}
\dot{\hat{x}}_{21} \\
\dot{\hat{x}}_{22}
\end{array}\right]=} & {\left[\begin{array}{cc}
-2 & 0 \\
0 & -3
\end{array}\right]\left[\begin{array}{l}
\hat{x}_{21} \\
\hat{x}_{22}
\end{array}\right] } \\
& +\beta_{22}\left[\begin{array}{l}
1 \\
1
\end{array}\right]\left(y_{2}-\widehat{x}_{21}-\widehat{x}_{22}\right) \beta_{m 22}, \\
\dot{\beta}_{22}= & \left\|y_{2}-\widehat{x}_{21}-\widehat{x}_{22}\right\|^{2} \beta_{m 22}-\beta_{22}+\beta_{22}^{*}, \\
\beta_{m 22}= & 3+y_{2}^{2}+y_{2}^{4}+\left\|\beta_{21}\left(\hat{x}_{21}+\widehat{x}_{22}\right) \beta_{m 21}\right\|^{2} .
\end{aligned}
$$

In the simulation, one takes $\theta_{11}=\theta_{12}=\theta_{13}=1, \theta_{21}=\theta_{22}=$ $\theta_{23}=-1, d_{1}=1, d_{2}=1+\sin (t), x_{11}(0)=1, x_{12}(0)=$ $-1, x_{21}(0)=1, x_{22}(0)=-1, \omega_{1}(0)=\omega_{2}(0)=0, \widehat{x}_{11}(0)=$ $\widehat{x}_{12}(0)=\widehat{x}_{21}(0)=\widehat{x}_{22}(0)=0$, and $\beta_{11}(0)=\beta_{12}(0)=$ $\beta_{21}(0)=\beta_{22}(0)=0.1$.

First, choosing $\beta_{11}^{*}=0.8, \beta_{12}^{*}=1, \beta_{21}^{*}=1.2$, and $\beta_{22}^{*}=$ 1.4 , we obtained the simulation results as showed in Figures $1-3$. It can be seen that all the signals are bounded, but the system states $x$ and the observer errors $e$ are not very small.

Under the same initial conditions, taking $\beta_{11}^{*}=80, \beta_{12}^{*}=$ $100, \beta_{21}^{*}=120$, and $\beta_{22}^{*}=140$, we obtained the simulation results as showed in Figures $4-6$. It can be seen that all the signals are bounded and the system states $x$ and the observer errors $e$ are very small now.

\section{Conclusion}

In this paper, based on adaptive nonlinear damping, a novel decentralized robust adaptive output feedback stabilization is proposed for a large-scale interconnected nonlinear system with general uncertainties. In each subsystem, the proposed stabilization only has two adaptive parameters, and it is not needed to generate an additional dynamic signal or estimate the unknown parameters no matter how high the order of the unmodeled dynamics is and how many unknown parameters there are. Under certain assumptions, the proposed scheme 
guarantees that all the dynamic signals in the interconnected nonlinear system are bounded. Furthermore, the system states and estimate errors can be made arbitrarily small by choosing the design parameters appropriately large. Finally, simulation results illustrated the effectiveness of the proposed scheme.

\section{Competing Interests}

The authors declare that there is no conflict of interests regarding the publication of this paper.

\section{Acknowledgments}

The authors would like to acknowledge the funding received from the Scientific Research Fund of Sichuan Provincial Science \& Technology Department (under Grants no. 2014GZ0009, no. 2015JY025, and no. 2015GZ0304), the Scientific Research Fund of Sichuan Provincial Education Department (under Grants no. 14ZA0171 and no. 16ZA0206), the Fund of Robot Technology Used for Special Environment Key Laboratory of Sichuan Province (no. 14zxtk04), the State Key Laboratory of Robotics, Shenyang Institute of Automation, Chinese Academy of Sciences (no. 2014-009), and the Project Supported by the Scientific Research Foundation of CUIT (nos. KYTZ201410, J201508, and KYTZ201625) to conduct this research investigation.

\section{References}

[1] D.-Z. Zheng, "Decentralized output feedback stabilization of a class of nonlinear interconnected systems," IEEE Transactions on Automatic Control, vol. 34, no. 12, pp. 1297-1300, 1989.

[2] D. T. Gavel and D. D. Siljak, "Decentralized adaptive control: structural conditions for stability," IEEE Transactions on Automatic Control, vol. 34, no. 4, pp. 413-426, 1989.

[3] Y. Liu and X.-Y. Li, "Decentralized robust adaptive control of nonlinear systems with unmodeled dynamics," IEEE Transactions on Automatic Control, vol. 47, no. 5, pp. 848-856, 2002.

[4] Q. H. Wu, L. Jiang, and J. Y. Wen, "Decentralized adaptive control of interconnected non-linear systems using high gain observer," International Journal of Control, vol. 77, no. 8, pp. 703712, 2004

[5] N. Hovakimyan, E. Lavretsky, A. Calise, and R. Sattigeri, "Decentralized adaptive output feedback control via input/output inversion," International Journal of Control, vol. 79, no. 12, pp. 1538-1551, 2006.

[6] S. S. Stankovic, D. M. Stipanovic, and D. D. Siljak, "Decentralized dynamic output feedback for robust stabilization of a class of nonlinear interconnected systems," Automatica, vol. 43, no. 5, pp. 861-867, 2007.

[7] A. Swarnakar, H. J. Marquez, and T. Chen, "A new scheme on robust observer-based control design for interconnected systems with application to an industrial utility boiler," IEEE Transactions on Control Systems Technology, vol. 16, no. 3, pp. 539-547, 2008.

[8] S. Stanković and D. D. Šiljak, "Robust stabilization of nonlinear interconnected systems by decentralized dynamic output feedback," Systems \& Control Letters, vol. 58, no. 4, pp. 271-275, 2009.
[9] K. Kalsi, J. Lian, and S. H. Zak, "Decentralized dynamic output feedback control of nonlinear interconnected systems," IEEE Transactions on Automatic Control, vol. 55, no. 8, pp. 1964-1970, 2010.

[10] C. Hua and X. Guan, "Output feedback stabilization for timedelay nonlinear interconnected systems using neural networks," IEEE Transactions on Neural Networks, vol. 19, no. 4, pp. 673$688,2008$.

[11] S. Mehraeen, S. Jagannathan, and M. L. Crow, "Decentralized dynamic surface control of large-scale interconnected systems in strict-feedback form using neural networks with asymptotic stabilization," IEEE Transactions on Neural Networks, vol. 22, no. 11, pp. 1709-1722, 2011.

[12] H. Wu, "Decentralized adaptive robust control for a class of large scale systems with uncertainties in the interconnections," International Journal of Control, vol. 76, no. 3, pp. 253-265, 2003.

[13] H. Wu, "Decentralised adaptive robust control of uncertain large-scale non-linear dynamical systems with time-varying delays," IET Control Theory \& Applications, vol. 6, no. 5, pp. 629640, 2012.

[14] J. Lavaei, "Decentralized implementation of centralized controllers for interconnected systems," IEEE Transactions on Automatic Control, vol. 57, no. 7, pp. 1860-1865, 2012.

[15] H. Fan, L. Han, C. Wen, and L. Xu, "Decentralized adaptive output-feedback controller design for stochastic nonlinear interconnected systems," Automatica, vol. 48, no. 11, pp. 28662873, 2012.

[16] D. Liu, D. Wang, and H. Li, "Decentralized stabilization for a class of continuous-time nonlinear interconnected systems using online learning optimal control approach," IEEE Transactions on Neural Networks and Learning Systems, vol. 25, no. 2, pp. 418-428, 2014.

[17] B. R. Barmish and A. R. Galimidi, "Robustness of luenberger observers: linear systems stabilized via nonlinear control," Automatica, vol. 22, no. 4, pp. 413-423, 1986.

[18] Z.-P. Jiang and L. Praly, "Design of robust adaptive controllers for nonlinear systems with dynamic uncertainties," Automatica, vol. 34, no. 7, pp. 825-840, 1998.

[19] Y. Liu, "Robust adaptive observer for nonlinear systems with unmodeled dynamics," Automatica, vol. 45, no. 8, pp. 1891-1895, 2009.

[20] Q. Yang and Y. Liu, "Adaptive state estimation of multi-input and multi-output non-linear systems with general uncertainties both in the state and output equations," IET Control Theory \& Applications, vol. 10, no. 3, pp. 354-362, 2016.

[21] H. K. Khalil, "Adaptive output feedback control of nonlinear systems represented by input-output models," IEEE Transactions on Automatic Control, vol. 41, no. 2, pp. 177-188, 1996.

[22] H. J. Sussmann and P. V. Kokotovic, "The peaking phenomenon and the global stabilization of nonlinear systems," IEEE Transactions on Automatic Control, vol. 36, no. 4, pp. 424-440, 1991. 


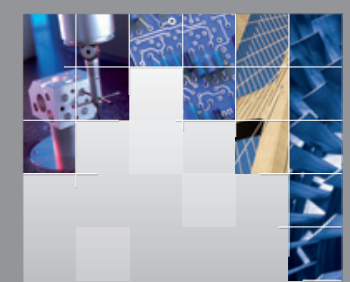

\section{Enfincering}
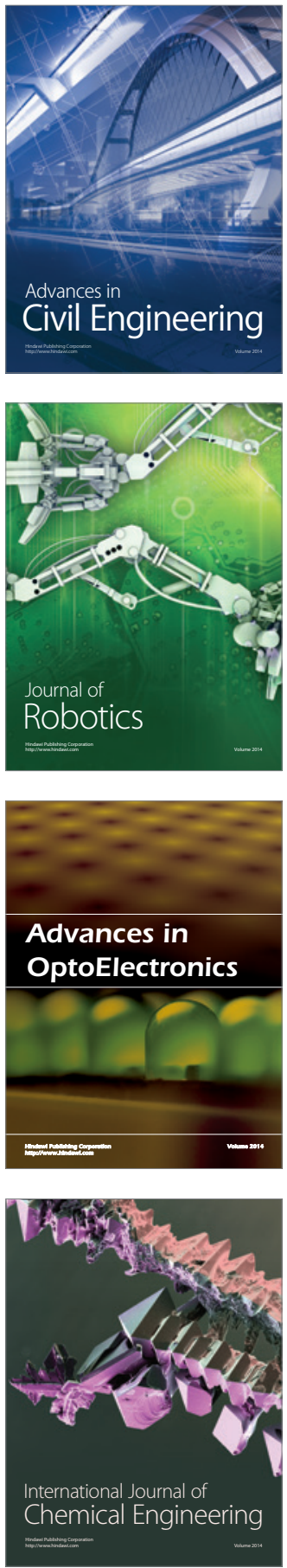

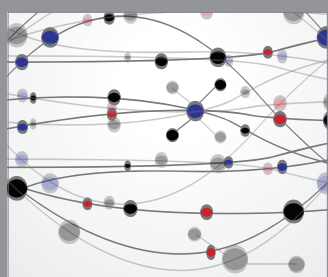

The Scientific World Journal

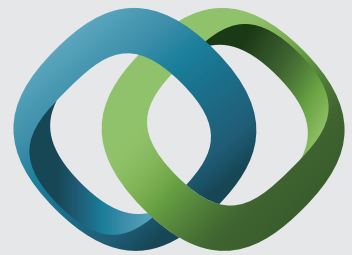

\section{Hindawi}

Submit your manuscripts at

http://www.hindawi.com
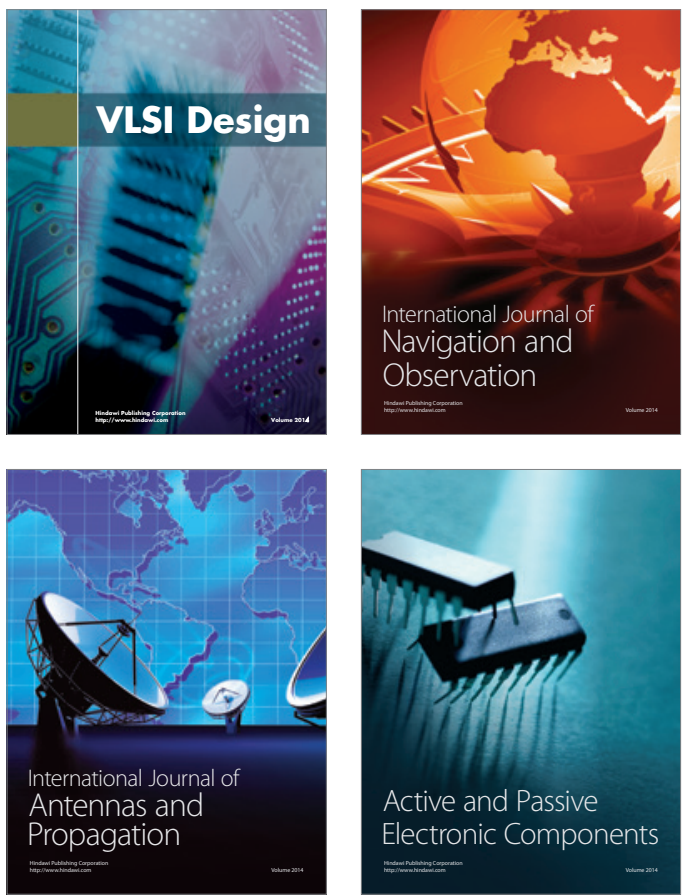
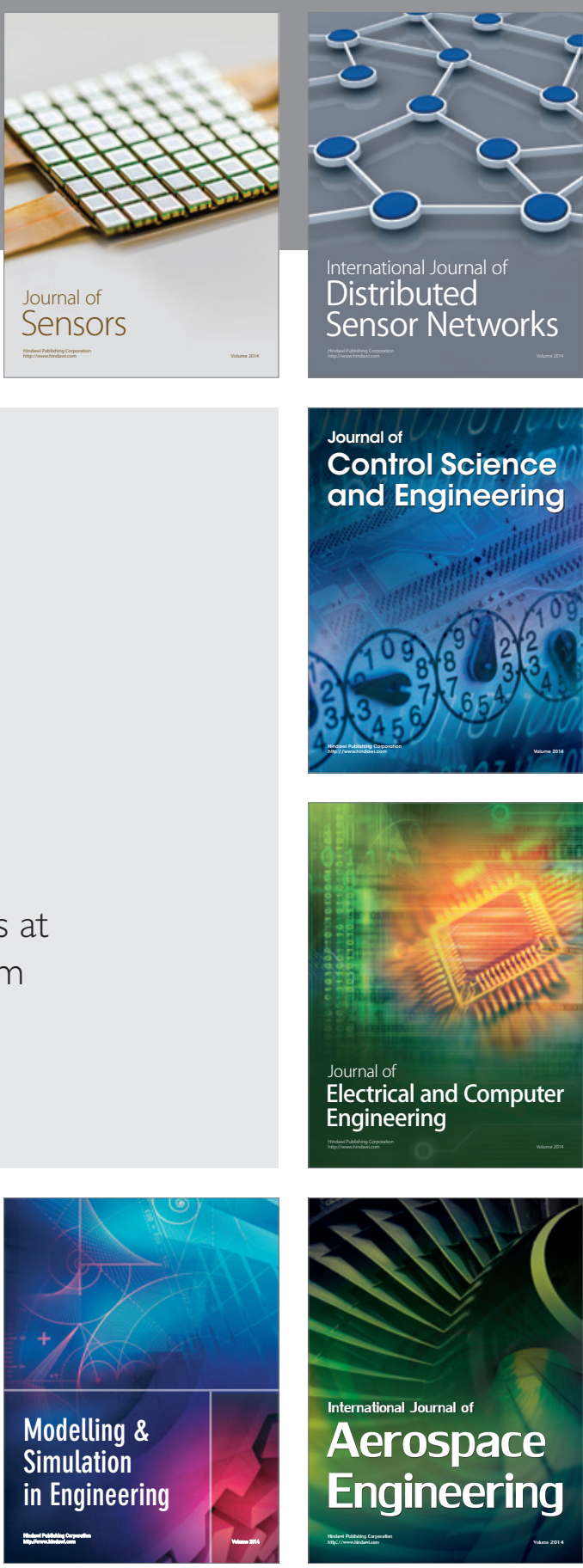

International Journal of

Distributed

Sensor Networks

Journal of

Control Science

and Engineering
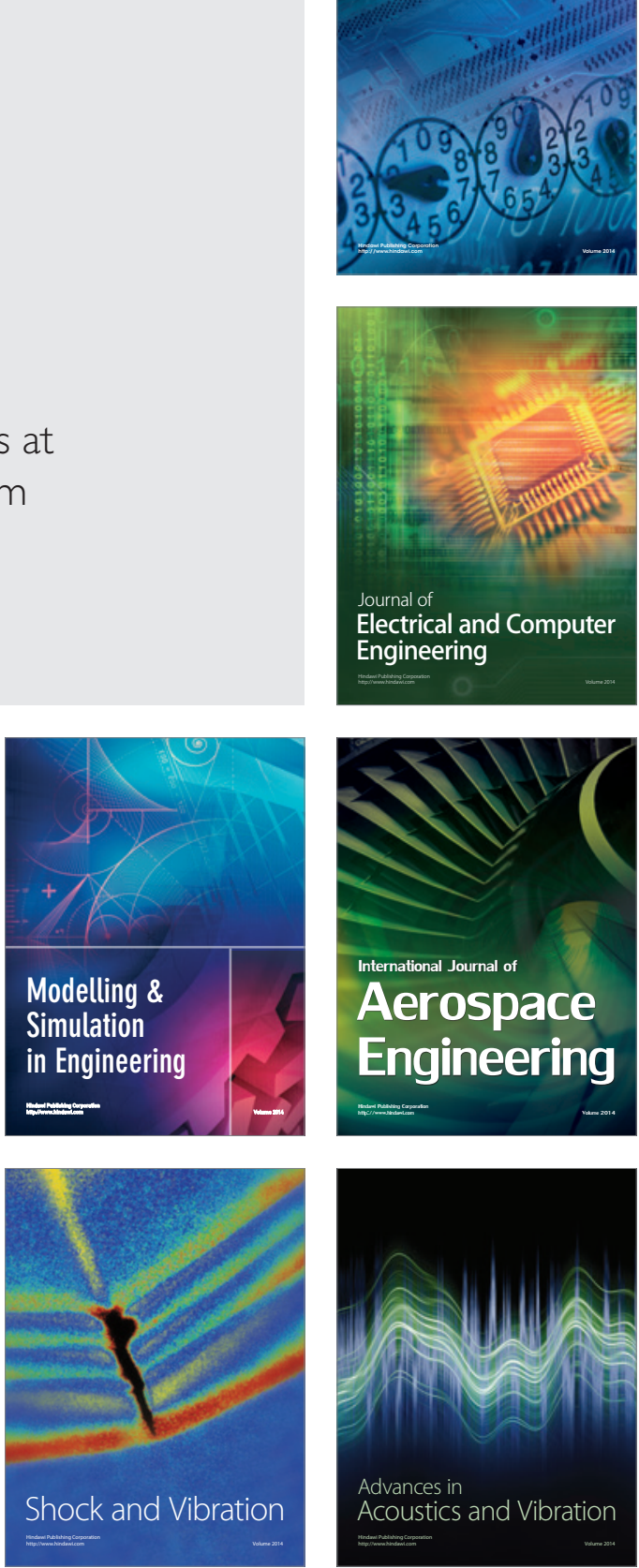